\title{
Targeting Trimeric and Tetrameric Proanthocyanidins from Cinnamomum verum Bark as Bioactives for Dental Therapies
}

Joo-Won Nam, ${ }^{\dagger,}$ Rasika S. Phansalkar, ${ }^{\dagger}$ David C. Lankin, ${ }^{\dagger}$ James B. McAlpine, ${ }^{\dagger, \dagger}$ Ariene A. Leme-Kraus, ${ }^{\S}$ Ana K. Bedran-Russo, ${ }^{\S, \perp}$ Shao-Nong Chen, ${ }^{\dagger, \ddagger}$ and Guido F. Pauli ${ }^{*},+$,

†Department of Pharmaceutical Sciences, Program for Collaborative Research in the Pharmaceutical Sciences (PCRPS), and Institute for Tuberculosis Research, College of Pharmacy, University of Illinois at Chicago, Chicago, IL 60612, United States

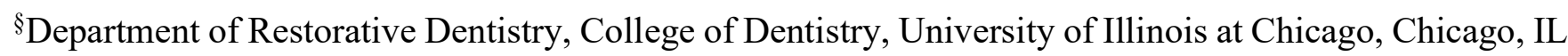
60612, United States

'College of Pharmacy, Yeungnam University, Gyeongsan, Gyeongbuk 712-749, Korea

${ }^{\perp}$ Department of General Dental Sciences, School of Dentistry, Marquette University, Milwaukee, WI 53233, United States 
S1. $\quad{ }^{1} \mathrm{H}$ NMR $\left(900 \mathrm{MHz}, \mathrm{CD}_{3} \mathrm{OD}, 298 \mathrm{~K}\right)$ of $\mathbf{1} \quad S 3$

S2. DEPTQ-135 NMR (225 MHz, CD $\left.{ }_{3} \mathrm{OD}, 298 \mathrm{~K}\right)$ of $\mathbf{1} \quad S 4$

S3. $\quad{ }^{1} \mathrm{H}-{ }^{1} \mathrm{H}$ COSY NMR $\left(\mathrm{CD}_{3} \mathrm{OD}, 298 \mathrm{~K}\right)$ of $\mathbf{1} \quad S 5$

S4. $\quad{ }^{1} \mathrm{H}-{ }^{1} \mathrm{H}$ ROESY NMR $\left(\mathrm{CD}_{3} \mathrm{OD}, 298 \mathrm{~K}\right)$ of $\mathbf{1} \quad S 6$

S5. $\quad{ }^{1} \mathrm{H}_{-}{ }^{13} \mathrm{C}$ HSQC NMR $\left(\mathrm{CD}_{3} \mathrm{OD}, 298 \mathrm{~K}\right)$ of $\mathbf{1} \quad S 8$

S6. $\quad{ }^{1} \mathrm{H}_{-}{ }^{13} \mathrm{C}$ HMBC NMR $\left(\mathrm{CD}_{3} \mathrm{OD}, 298 \mathrm{~K}\right)$ of $\mathbf{1} \quad S 9$

S7. $\quad{ }^{1} \mathrm{H}$ NMR $\left(900 \mathrm{MHz}, \mathrm{CD}_{3} \mathrm{OD}, 298 \mathrm{~K}\right)$ of $2 \quad S 10$

S8. $\quad$ DEPTQ-135 NMR (225 MHz, $\left.\mathrm{CD}_{3} \mathrm{OD}, 298 \mathrm{~K}\right)$ of $\mathbf{2} \quad S 11$

s9. $\quad{ }^{1} \mathrm{H}-{ }^{1} \mathrm{H}$ COSY NMR $\left(\mathrm{CD}_{3} \mathrm{OD}, 298 \mathrm{~K}\right)$ of $2 \quad S 12$

S10. $\quad{ }^{1} \mathrm{H}-{ }^{1} \mathrm{H}$ ROESY NMR $\left(\mathrm{CD}_{3} \mathrm{OD}, 298 \mathrm{~K}\right)$ of $2 \quad S 13$

S11. $\quad{ }^{1} \mathrm{H}_{-}{ }^{13} \mathrm{C}$ HSQC NMR $\left(\mathrm{CD}_{3} \mathrm{OD}, 298 \mathrm{~K}\right)$ of $2 \quad S 14$

S12 $\quad{ }^{1} \mathrm{H}-{ }^{13} \mathrm{C}$ HMBC NMR $\left(\mathrm{CD}_{3} \mathrm{OD}, 298 \mathrm{~K}\right)$ of $2 \quad S 15$

S13. $\quad{ }^{1} \mathrm{H}$ NMR (900 MHz, CD $\left.{ }_{3} \mathrm{OD}, 298 \mathrm{~K}\right)$ of $\mathbf{3} \quad S 16$

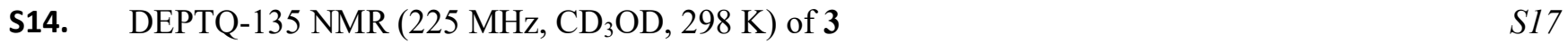

S15. $\quad{ }^{1} \mathrm{H}-{ }^{1} \mathrm{H}$ COSY NMR $\left(\mathrm{CD}_{3} \mathrm{OD}, 298 \mathrm{~K}\right)$ of $3 \quad S 18$

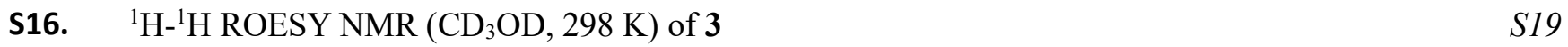

S17. $\quad{ }^{1} \mathrm{H}_{-}{ }^{13} \mathrm{C}$ HSQC NMR $\left(\mathrm{CD}_{3} \mathrm{OD}, 298 \mathrm{~K}\right)$ of $3 \quad S 20$

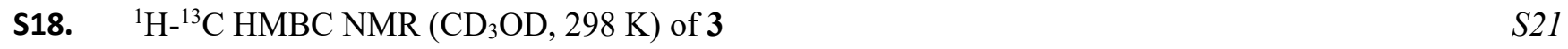

S19. The ${ }^{1} \mathrm{H}$ NMR fingerprint of 1 (PERCH .pms file format). $\quad S 22$

S20. The ${ }^{1} \mathrm{H}$ NMR fingerprint of 2 (PERCH .pms file format). $\quad S 23$

S21. The ${ }^{1} \mathrm{H}$ NMR fingerprint of the major conformer of $\mathbf{3}$ (PERCH .pms file format). S24

S22. The ${ }^{1} \mathrm{H}$ NMR fingerprint of the minor conformer of $\mathbf{3}$ (PERCH .pms file format).

S23. The ${ }^{1} \mathrm{H}$ NMR fingerprint of the mixture of $\mathbf{3}$ (PERCH .pms file format). $\quad S 26$

S24. The CD spectra of compounds 1-3 $\quad S 28$

S25. $\quad{ }^{1} \mathrm{H}$ Comparison of NMR spectra showing that deuterium exchange reaction occurred in $2 \quad S 29$

S26. Calibration of NMR temperature $\quad S 30$

S27. Calculation of the purity of $\mathbf{1}$ by qHNMR using 100\% method $S 31$

S28 Calculation of the purity of 2 by qHNMR using 100\% method $S 32$

S29 Calculation of the purity of $\mathbf{3}$ by qHNMR using 100\% method S33 
s1. ${ }^{1} \mathrm{H}$ NMR $\left(900 \mathrm{MHz}, \mathrm{CD}_{3} \mathrm{OD}, 298 \mathrm{~K}\right)$ spectrum of $\mathbf{1}$.
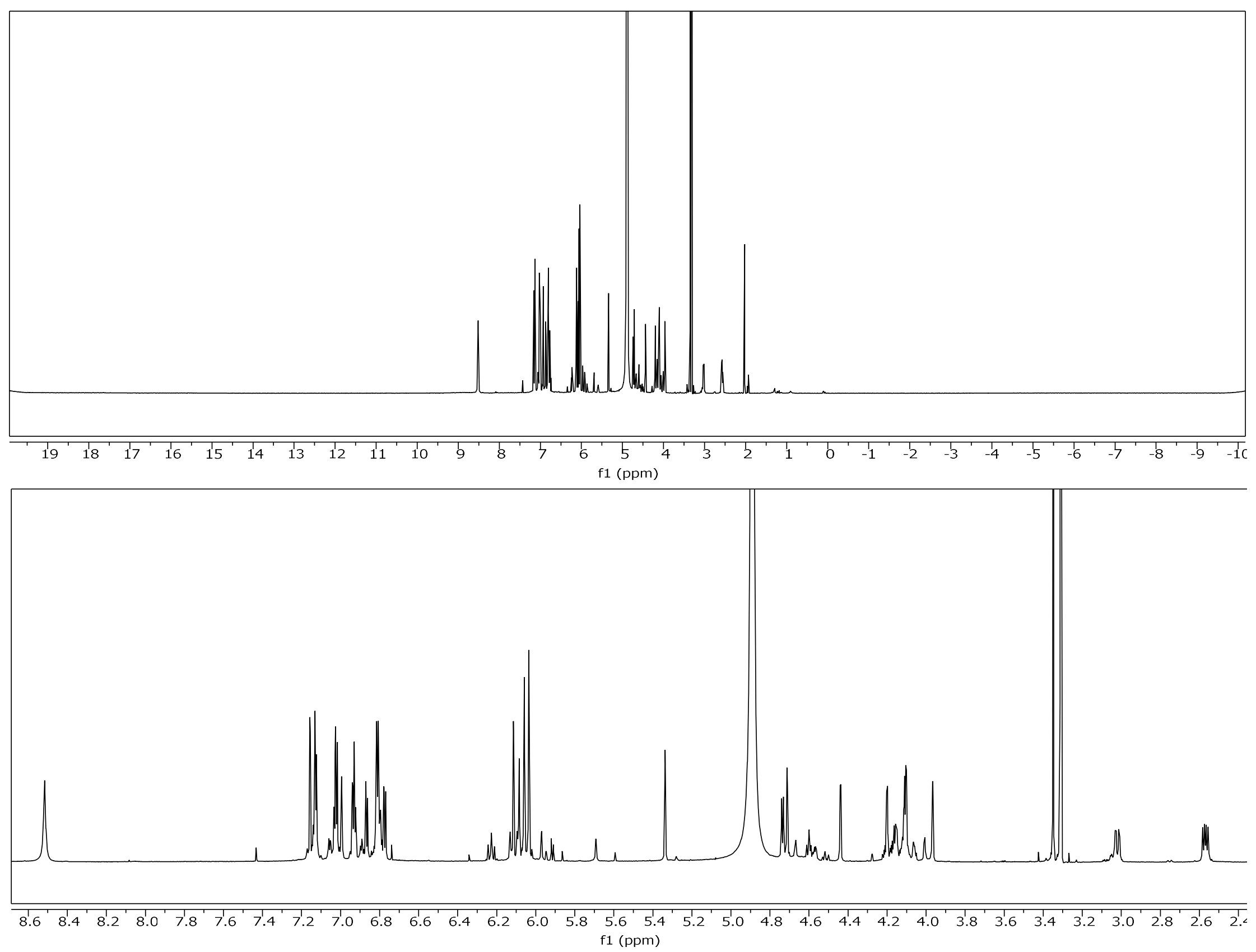
S2. DEPTQ-135 NMR (225 MHz, CD 3 OD, $298 \mathrm{~K})$ spectrum of $\mathbf{1}$.

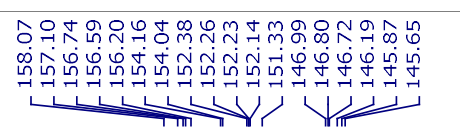

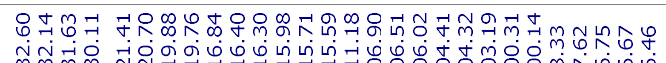

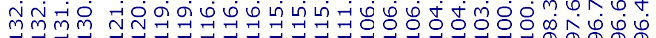
पा/

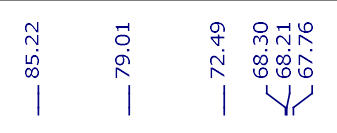

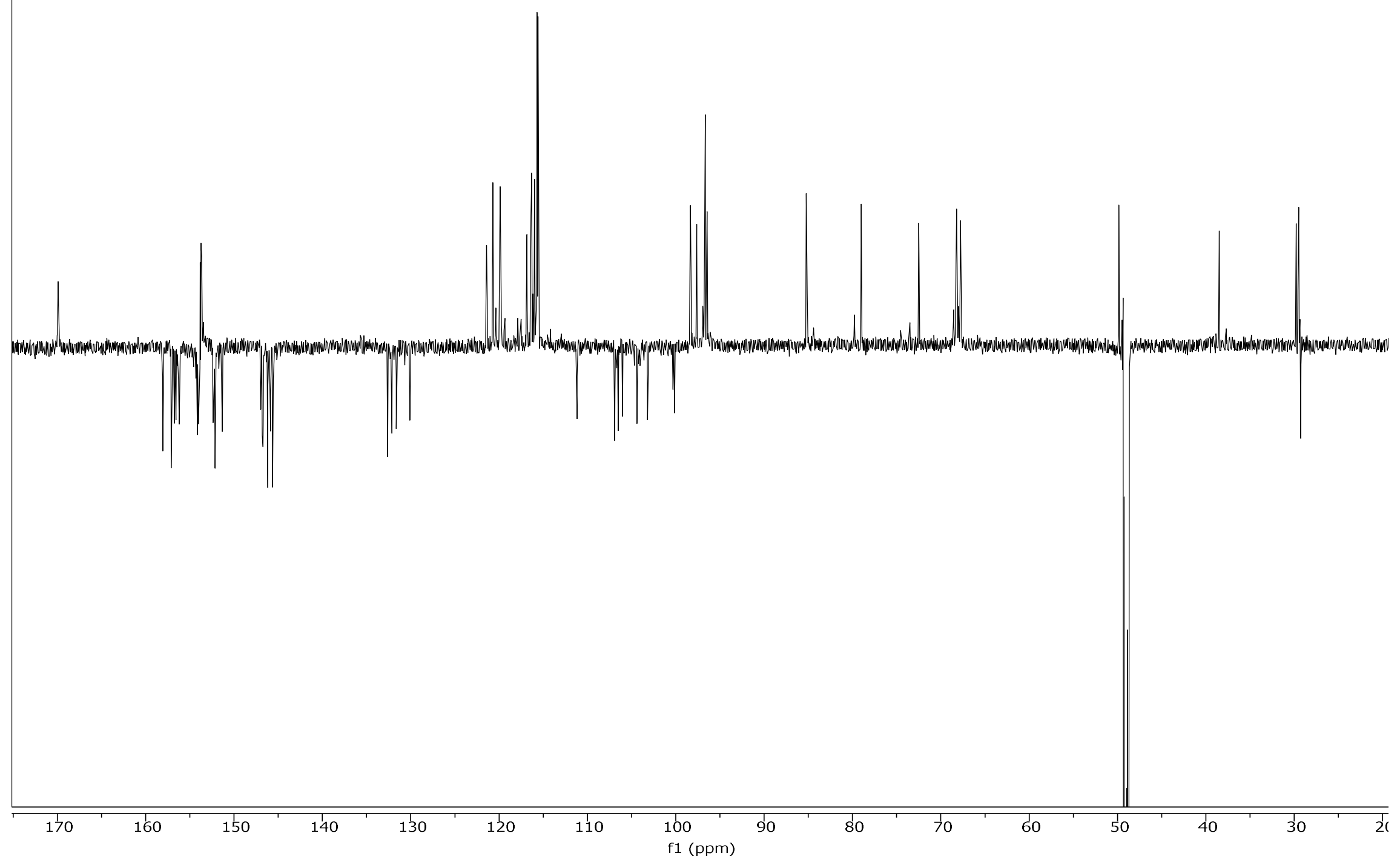


s3. ${ }^{1} \mathrm{H}-{ }^{1} \mathrm{H}$ COSY NMR spectrum $\left(\mathrm{CD}_{3} \mathrm{OD}, 298 \mathrm{~K}\right)$ of $\mathbf{1}$.

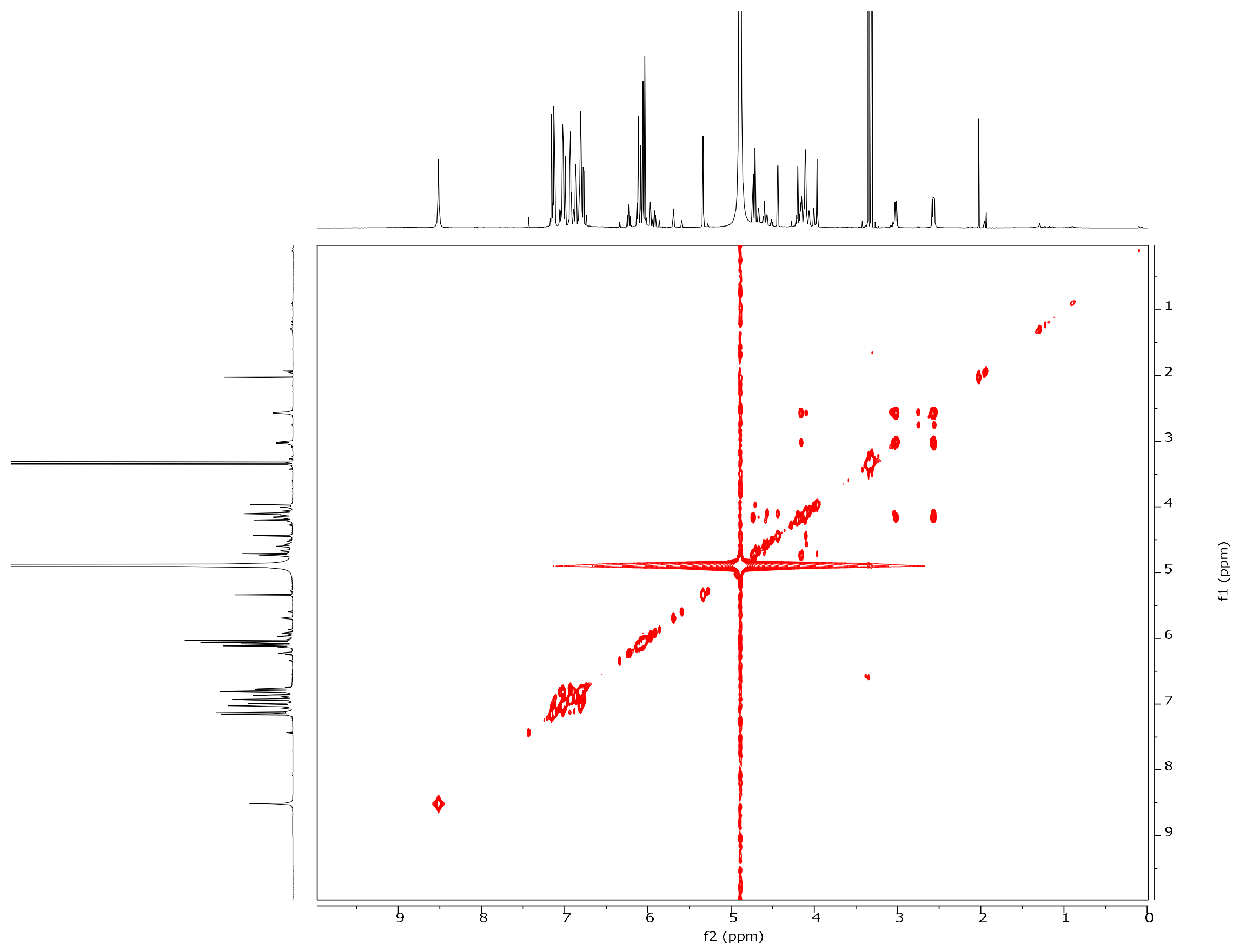


S4. $\quad{ }^{1} \mathrm{H}-{ }^{1} \mathrm{H}$ ROESY NMR spectrum $\left(\mathrm{CD}_{3} \mathrm{OD}, 298 \mathrm{~K}\right)$ of $\mathbf{1}$.

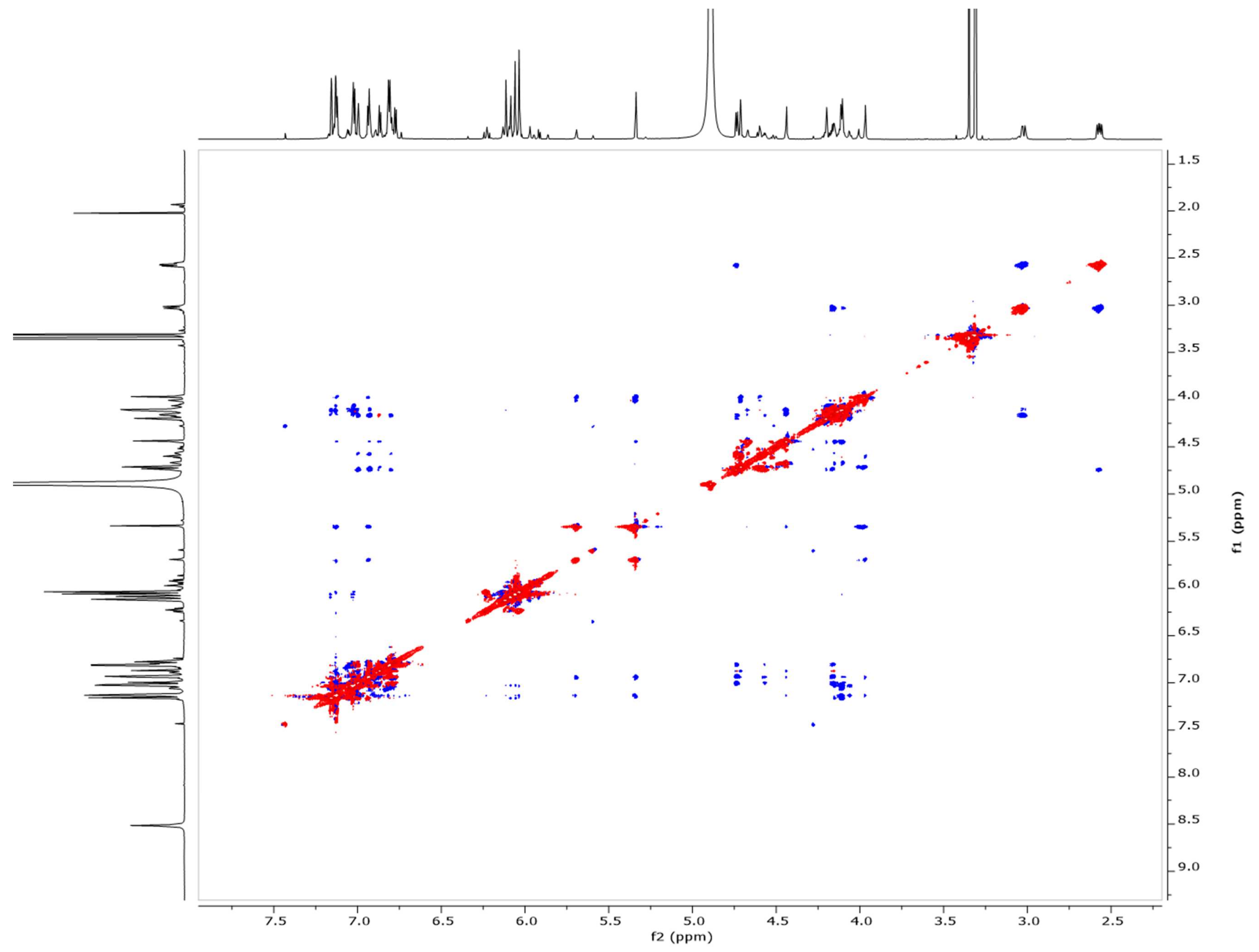


Expansion plots of the ${ }^{1} \mathrm{H}-{ }^{1} \mathrm{H}$ ROESY NMR spectrum of $\mathbf{1}$.
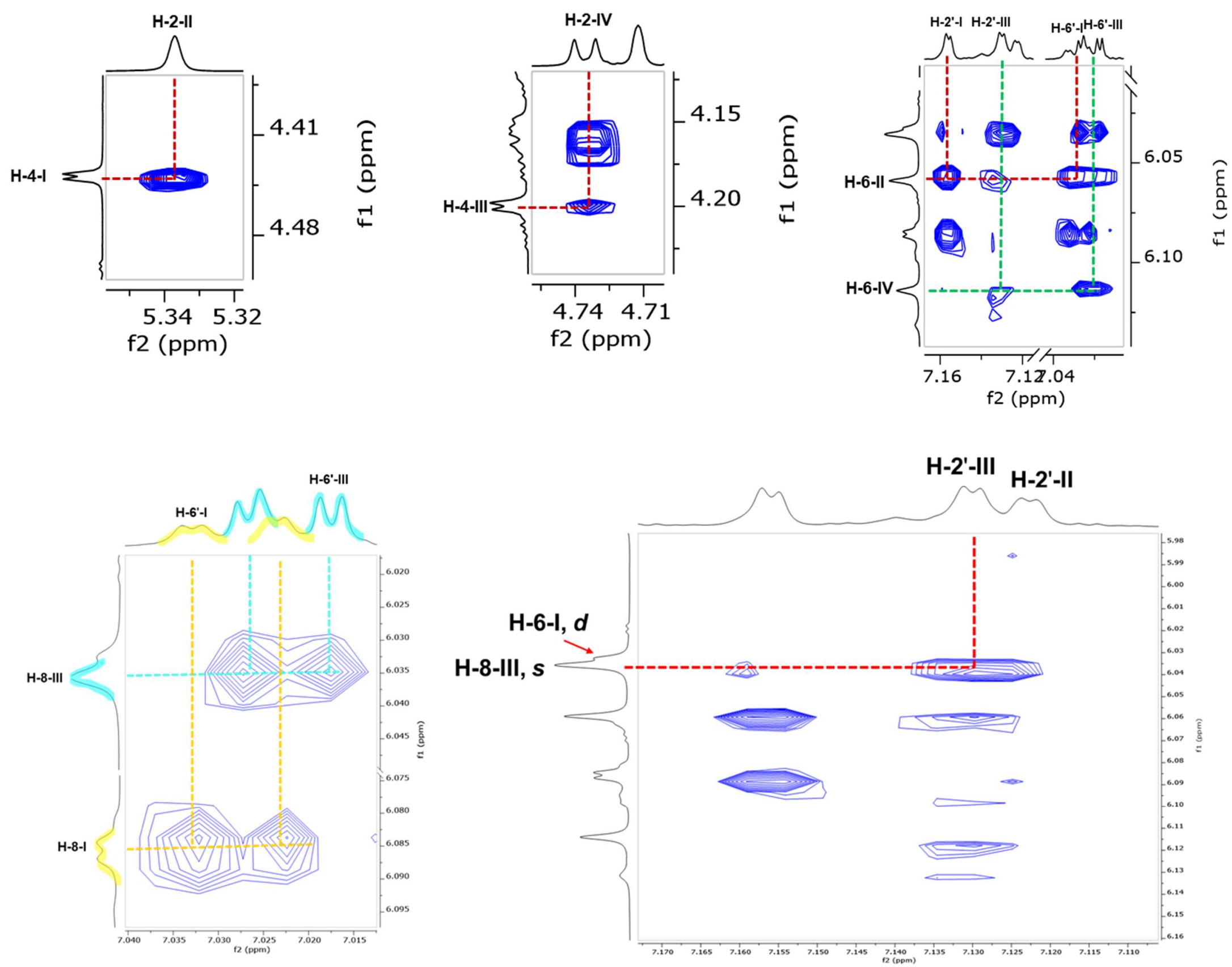
S5. $\quad{ }^{1} \mathrm{H}-{ }^{13} \mathrm{C}$ HSQC NMR spectrum $\left(\mathrm{CD}_{3} \mathrm{OD}, 298 \mathrm{~K}\right)$ of $\mathbf{1}$.

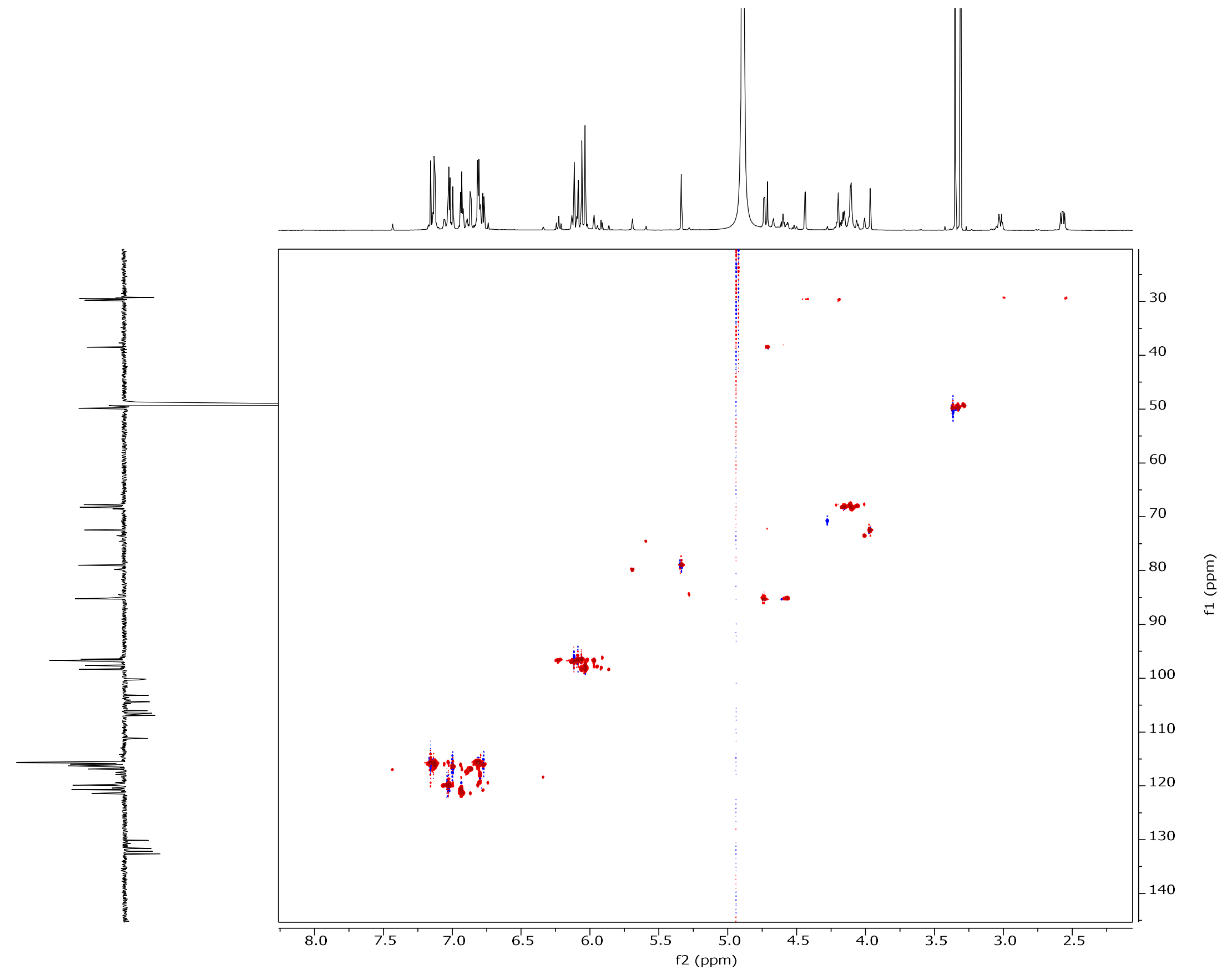


s6. $\quad{ }^{1} \mathrm{H}-{ }^{13} \mathrm{C}$ HMBC NMR spectrum $\left(\mathrm{CD}_{3} \mathrm{OD}, 298 \mathrm{~K}\right)$ of $\mathbf{1}$.

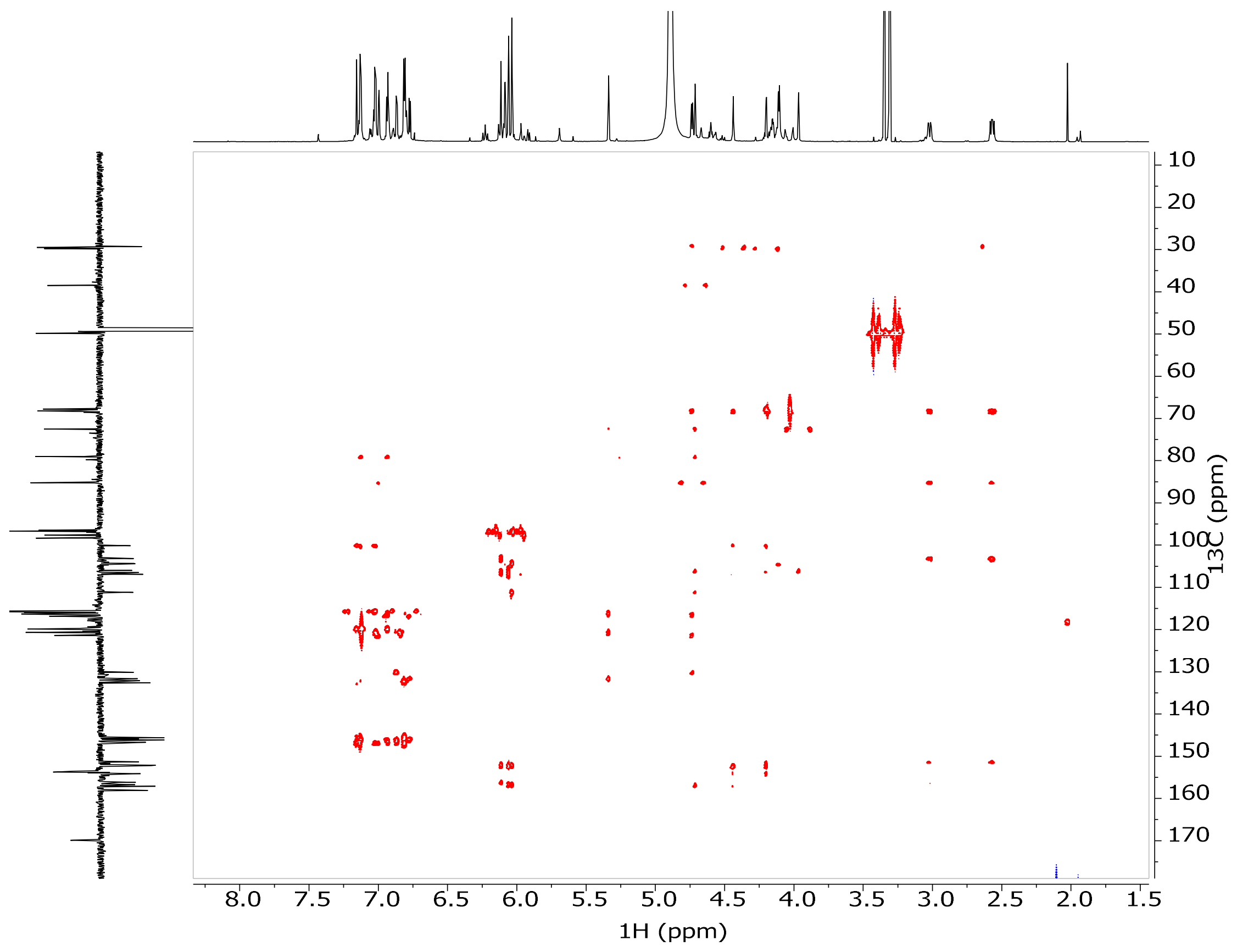


s7. $\quad{ }^{1} \mathrm{H}$ NMR spectrum $\left(900 \mathrm{MHz}, \mathrm{CD}_{3} \mathrm{OD}, 298 \mathrm{~K}\right)$ of 2.
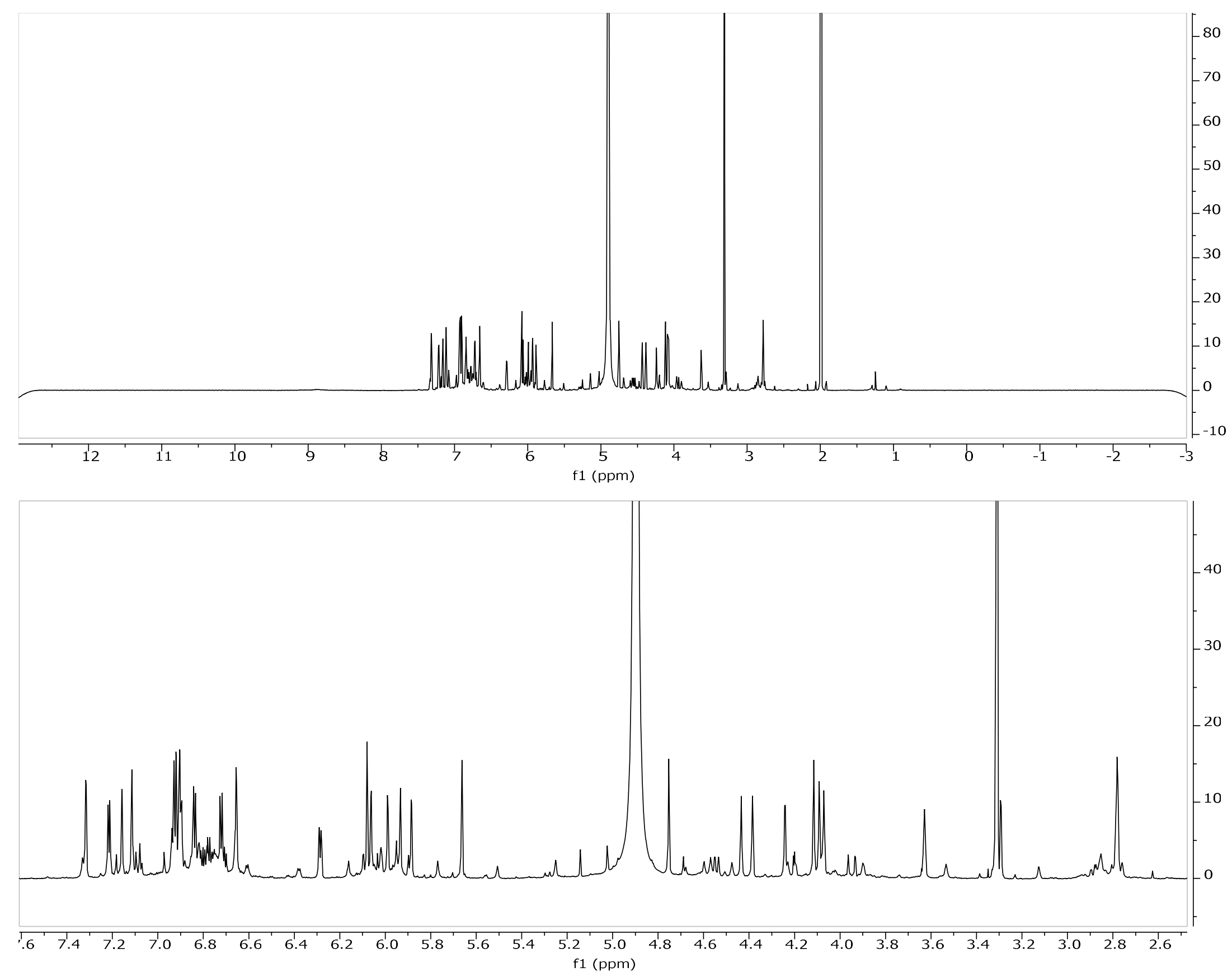
s8. DEPTQ-135 NMR spectrum (225 MHz, CD $\mathrm{OD}, 298 \mathrm{~K})$ of 2.

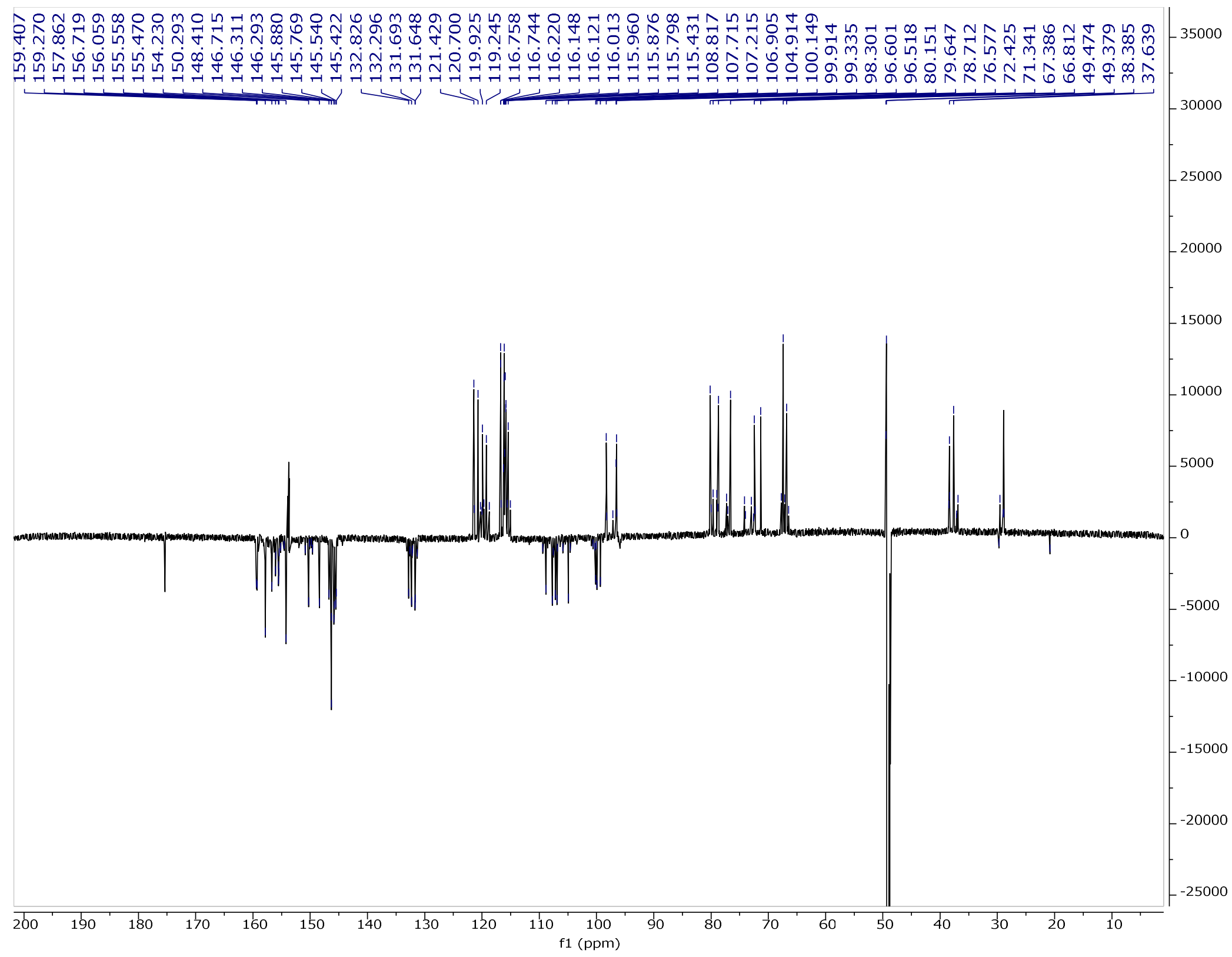


s9. $\quad{ }^{1} \mathrm{H}-{ }^{1} \mathrm{H}$ COSY NMR spectrum $\left(\mathrm{CD}_{3} \mathrm{OD}, 298 \mathrm{~K}\right)$ of 2 .

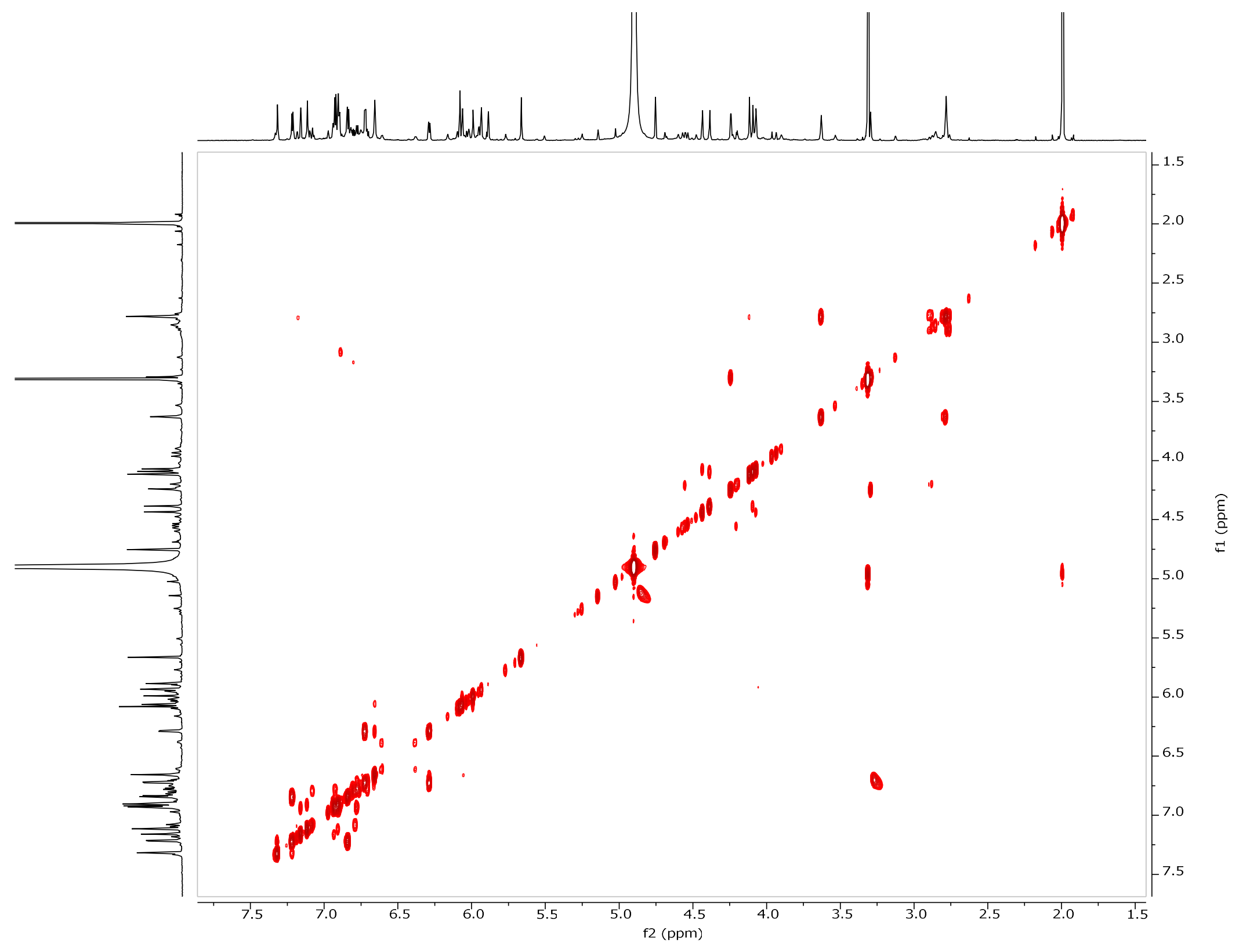


S10. $\quad{ }^{1} \mathrm{H}-{ }^{1} \mathrm{H}$ ROESY NMR spectrum $\left(\mathrm{CD}_{3} \mathrm{OD}, 298 \mathrm{~K}\right)$ of 2.

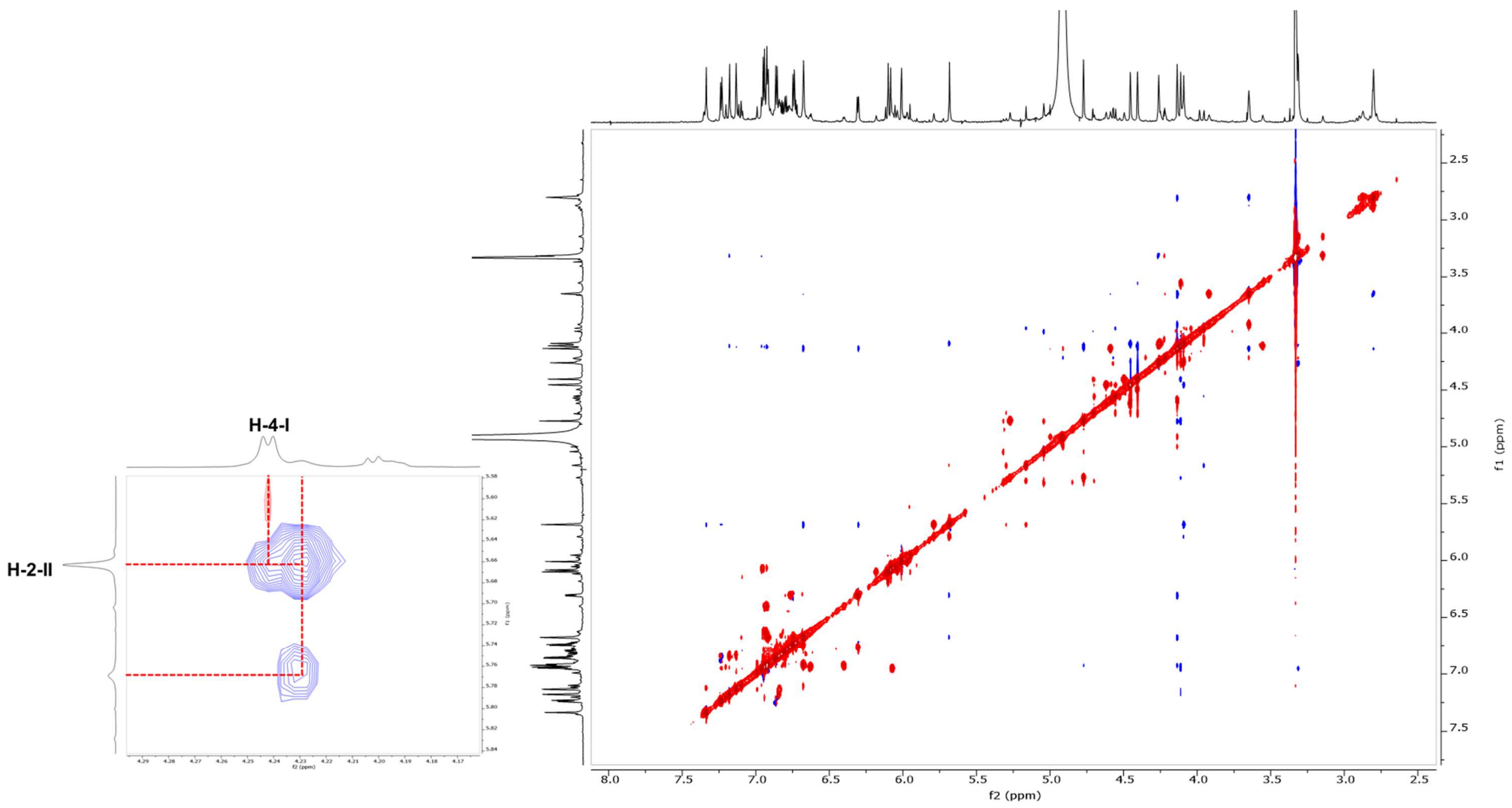

513 
S11. ${ }^{1} \mathrm{H}-{ }^{13} \mathrm{C}$ HSQC NMR spectrum $\left(\mathrm{CD}_{3} \mathrm{OD}, 298 \mathrm{~K}\right)$ of 2 .

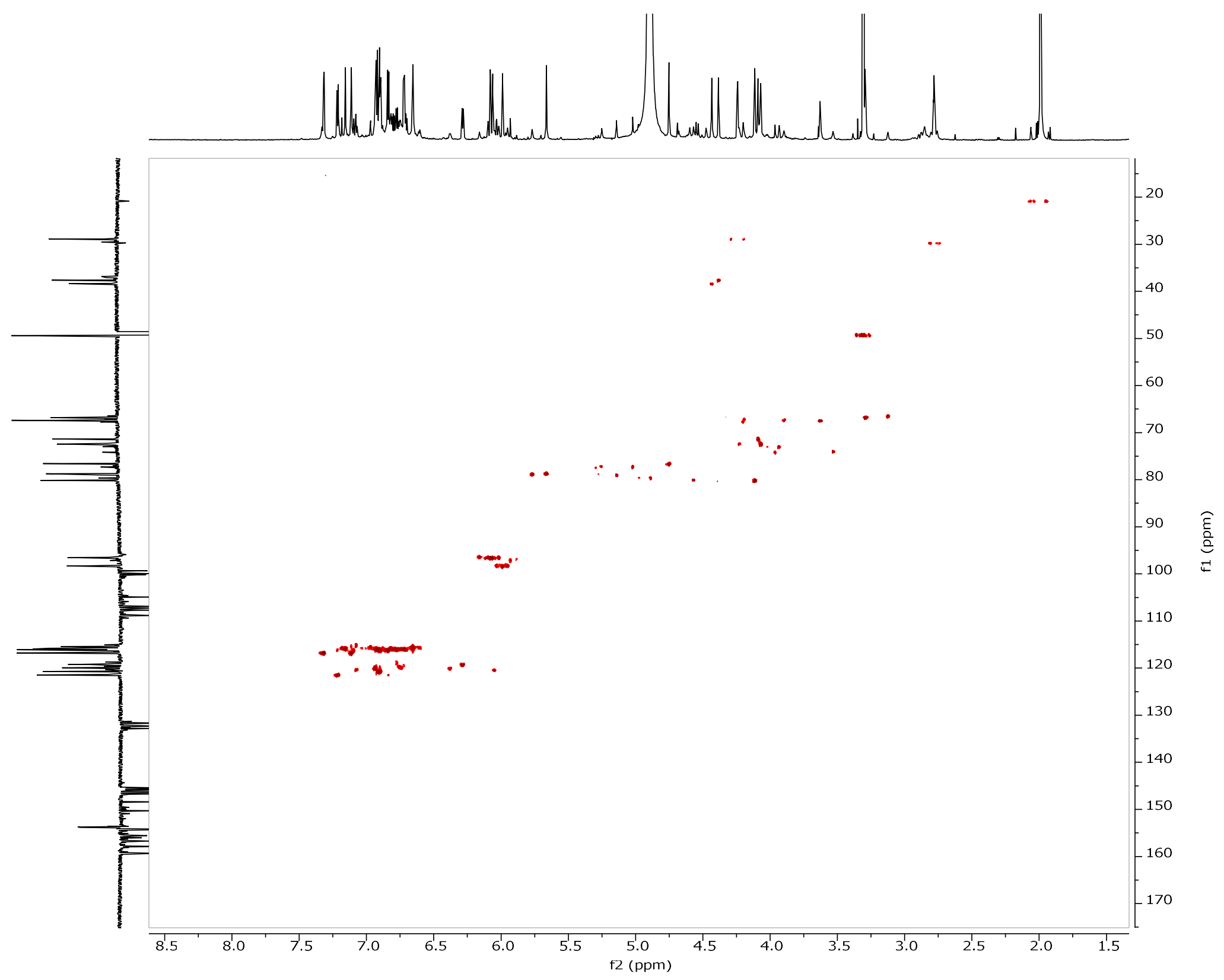


S12. ${ }^{1} \mathrm{H}-{ }^{13} \mathrm{C} \mathrm{HMBC}$ NMR spectrum $\left(\mathrm{CD}_{3} \mathrm{OD}, 298 \mathrm{~K}\right)$ of 2.

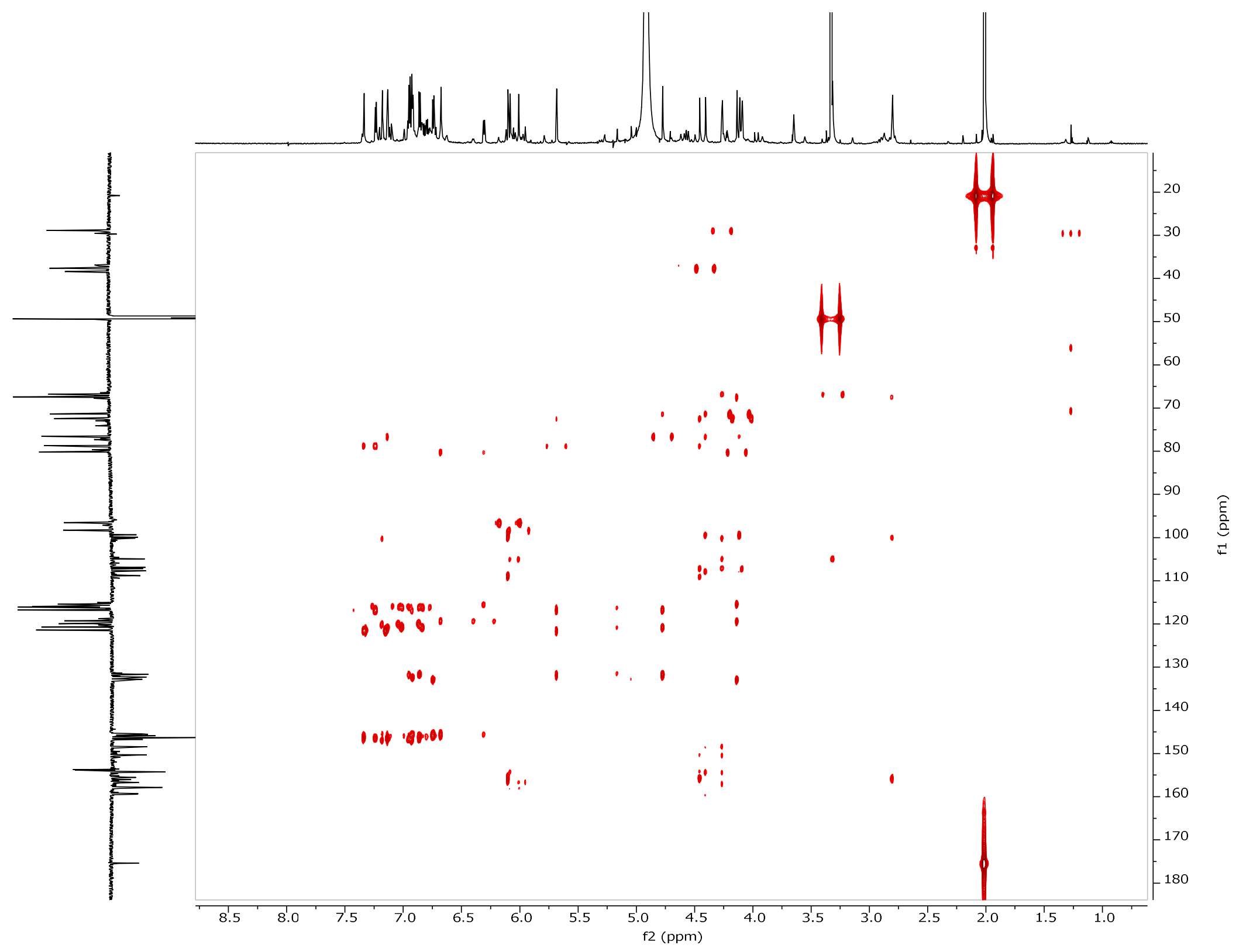


S13. ${ }^{1} \mathrm{H}$ NMR spectrum $\left(900 \mathrm{MHz}, \mathrm{CD}_{3} \mathrm{OD}, 298 \mathrm{~K}\right)$ of 3.

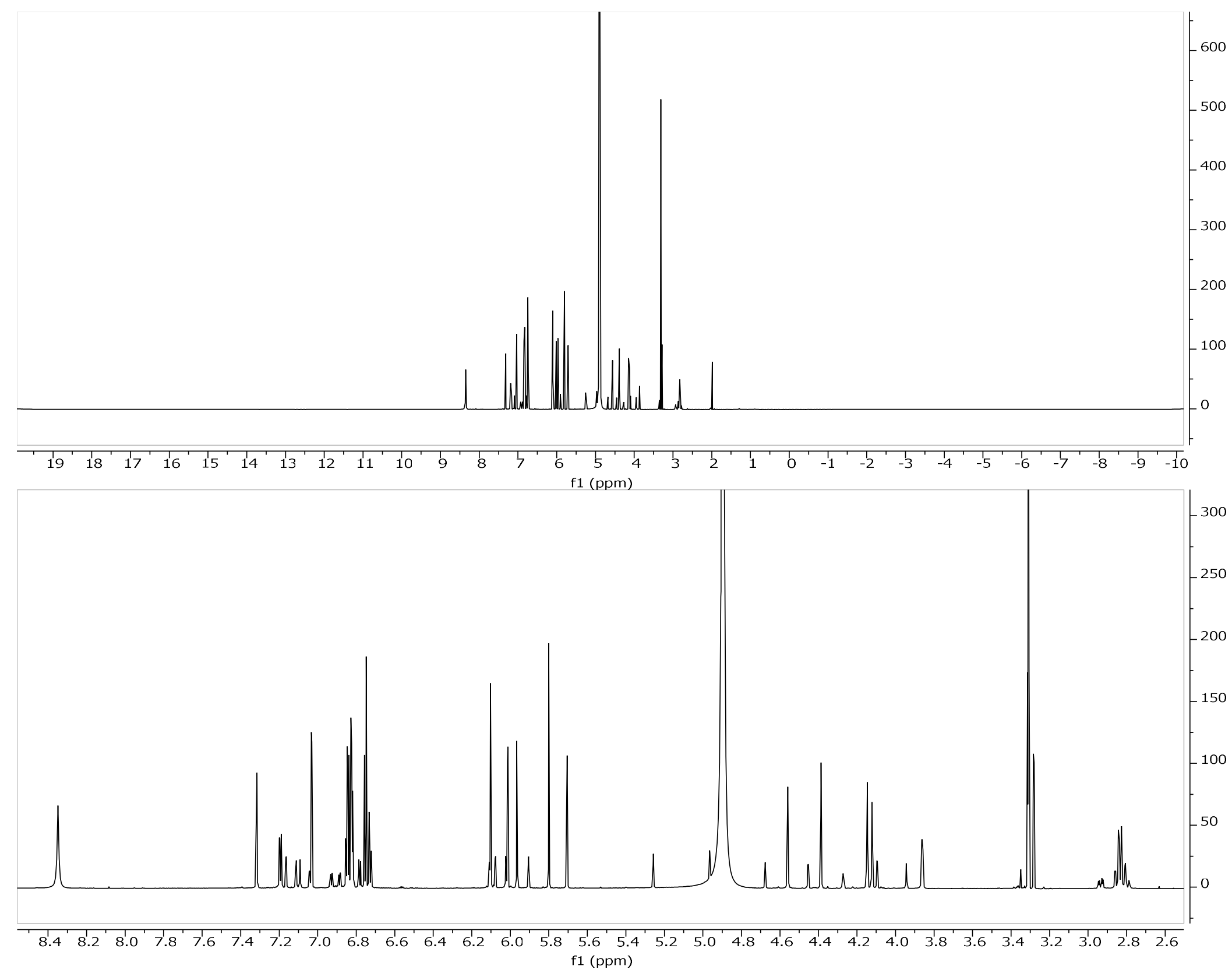


S14. DEPTQ-135 NMR spectrum (225 MHz, $\left.\mathrm{CD}_{3} \mathrm{OD}, 298 \mathrm{~K}\right)$ of 3.

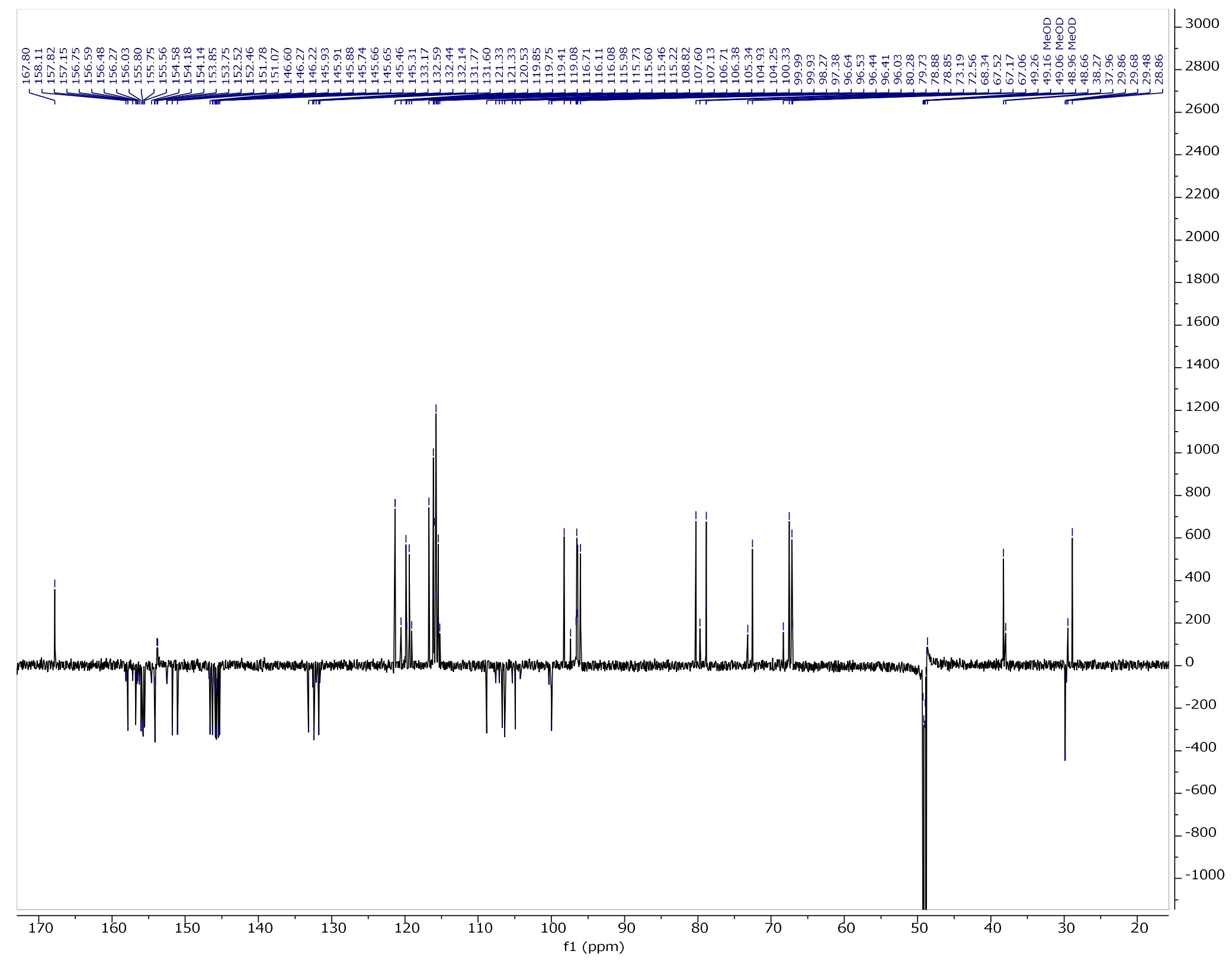


S15. ${ }^{1} \mathrm{H}-{ }^{1} \mathrm{H}$ COSY NMR spectrum $\left(\mathrm{CD}_{3} \mathrm{OD}, 298 \mathrm{~K}\right)$ of 3.

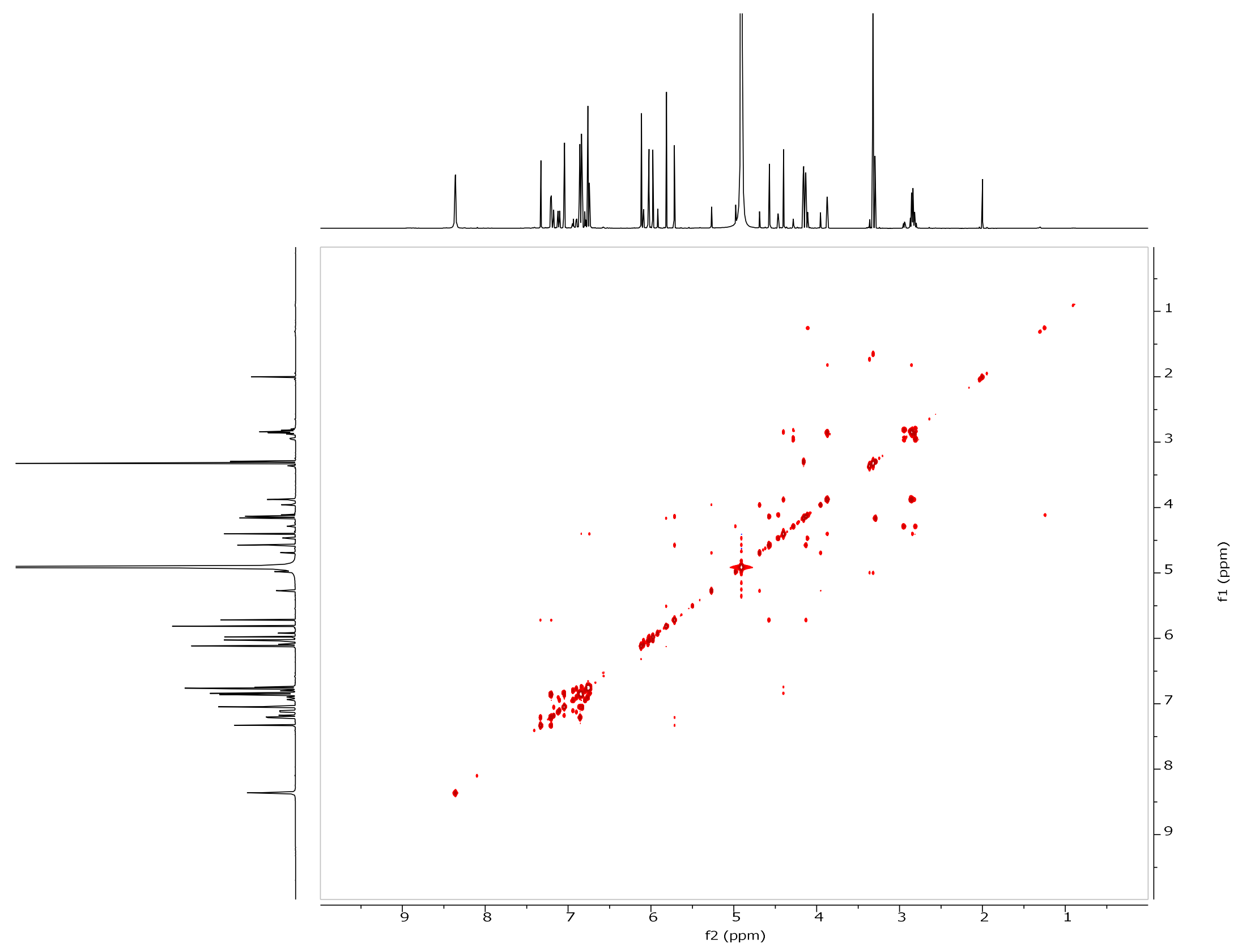


S16. $\quad{ }^{1} \mathrm{H}-{ }^{1} \mathrm{H}$ ROESY NMR spectrum $\left(\mathrm{CD}_{3} \mathrm{OD}, 298 \mathrm{~K}\right)$ of 3.

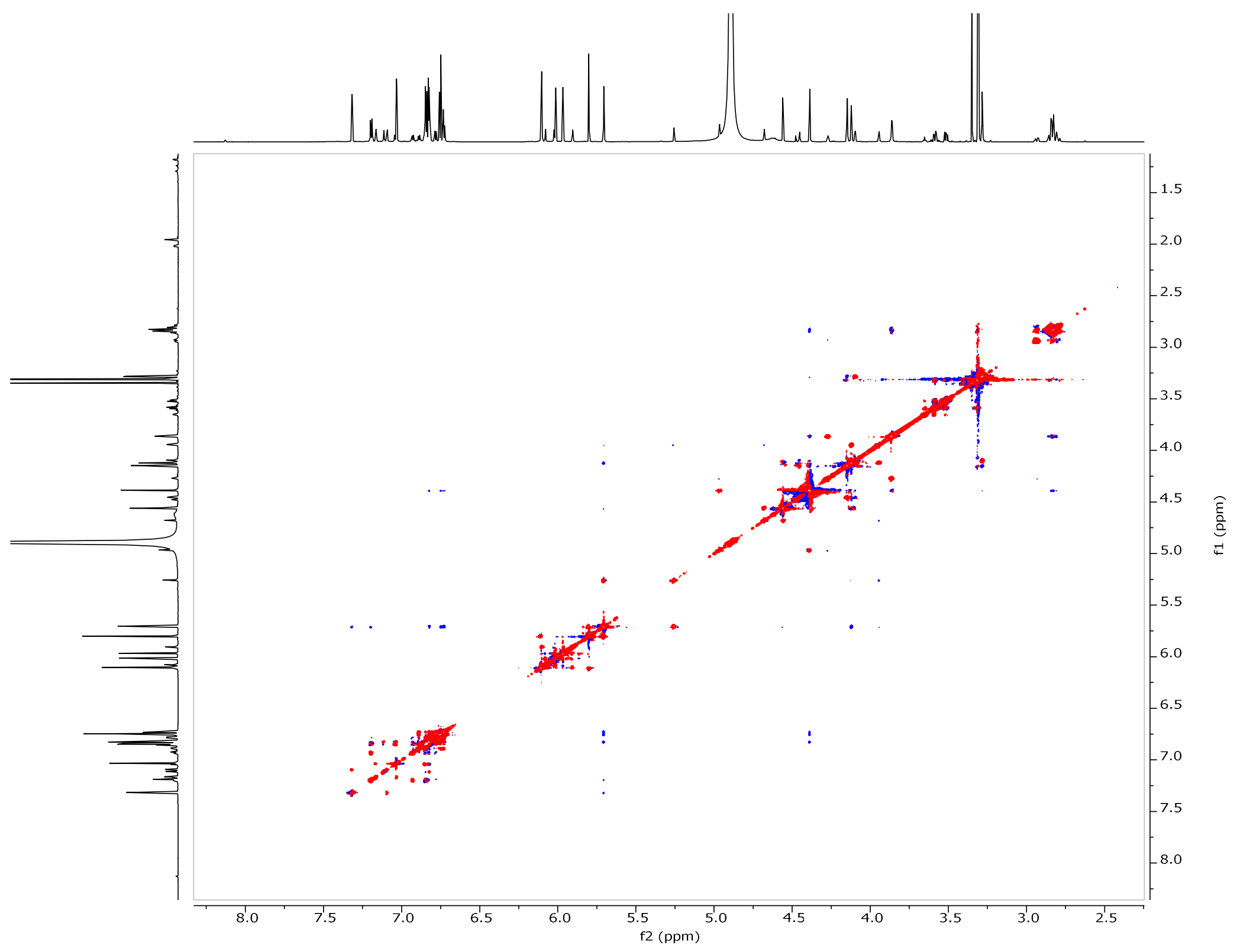


S17. $\quad{ }^{1} \mathrm{H}-{ }^{13} \mathrm{C}$ HSQC NMR spectrum $\left(\mathrm{CD}_{3} \mathrm{OD}, 298 \mathrm{~K}\right)$ of 3.

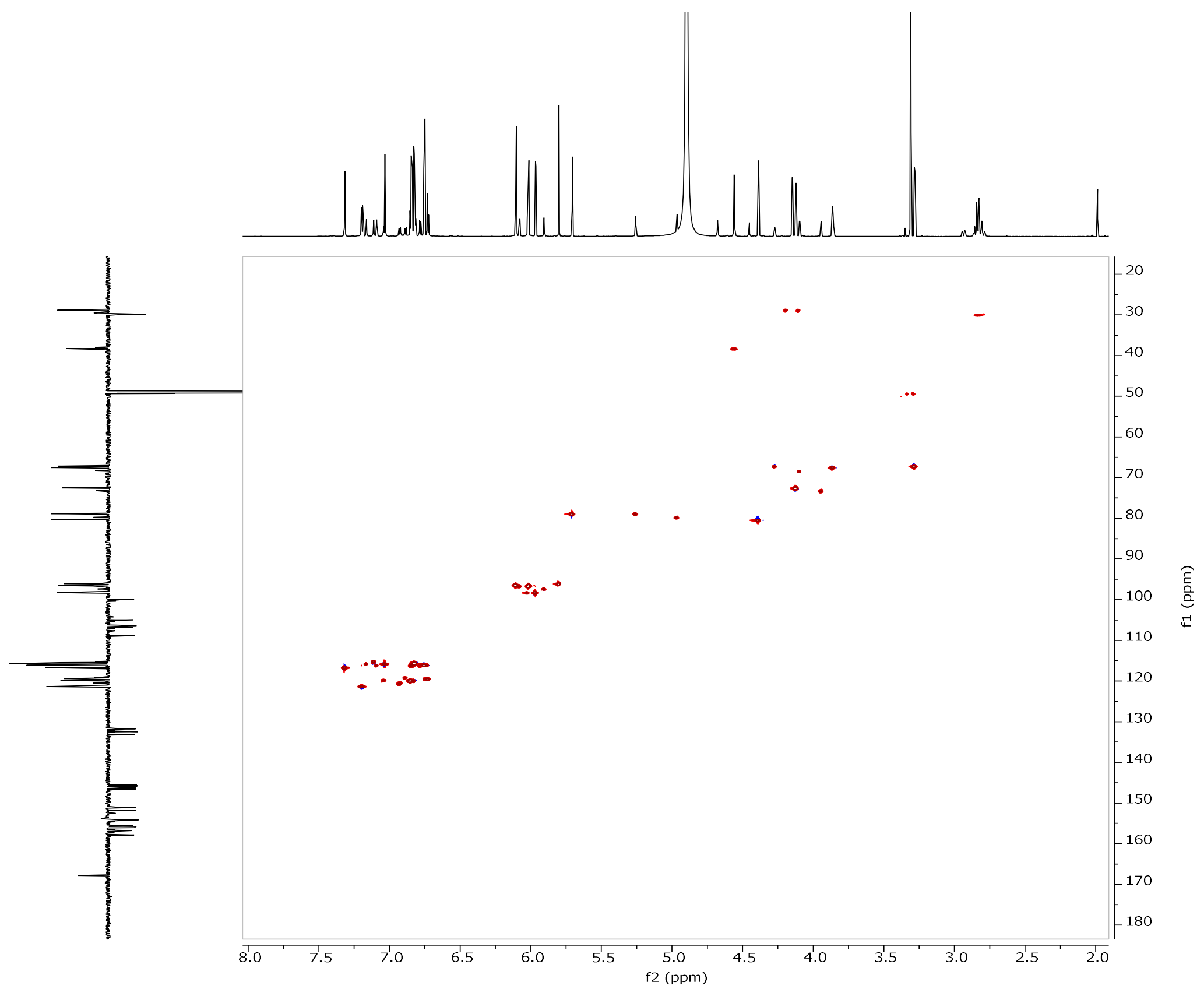


S18. $\quad{ }^{1} \mathrm{H}^{13} \mathrm{C}$ HMBC NMR spectrum $\left(\mathrm{CD}_{3} \mathrm{OD}, 298 \mathrm{~K}\right)$ of 3 .

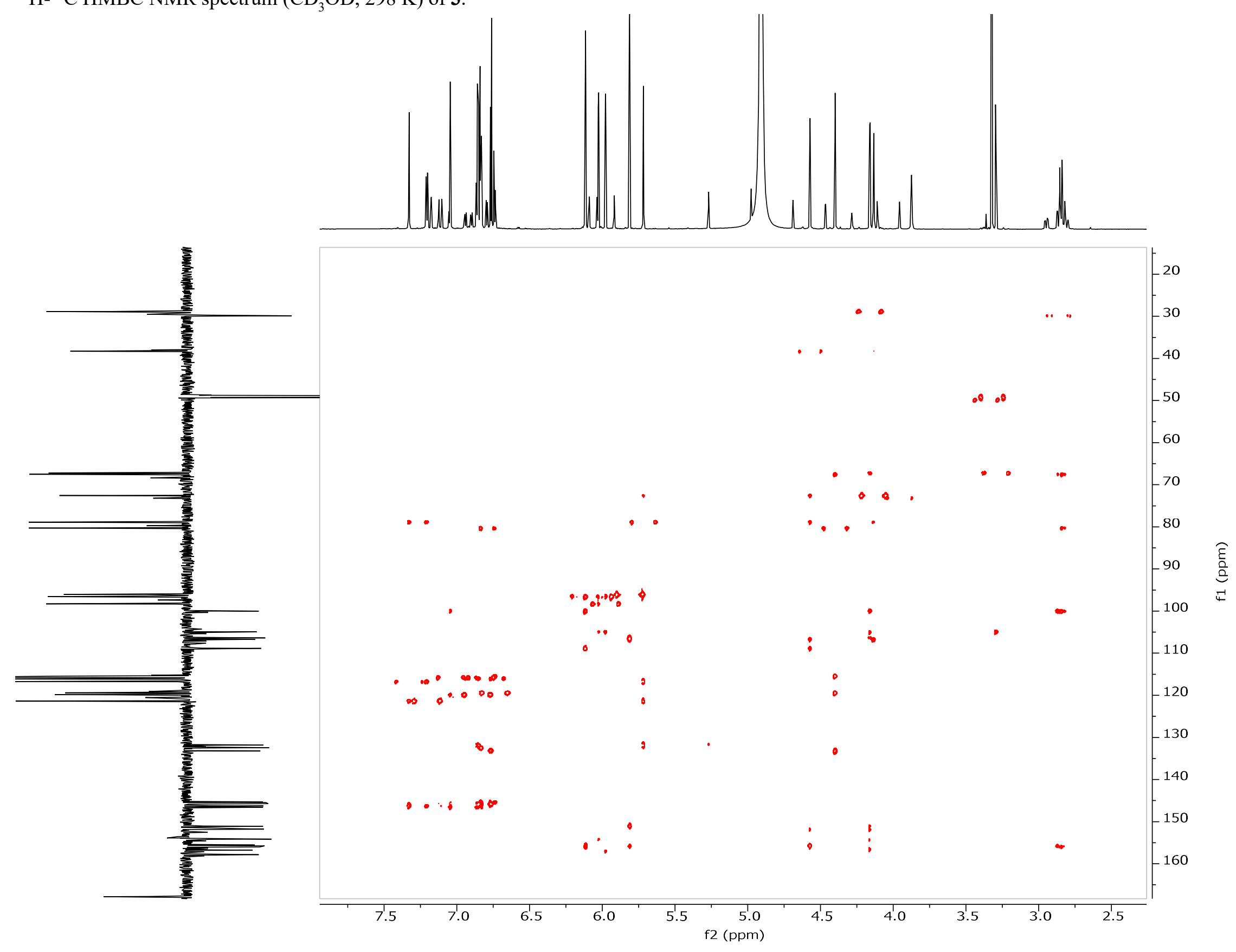


S19. The ${ }^{1} \mathrm{H}$ NMR fingerprint of $\mathbf{1}$ (PERCH .pms file format).

CHEMICAL SHIFTS(PPM):

PROTON $2 *$ SPIN $=1$ SPECIES $=1 \mathrm{H} \quad$ POPULATION $(\mathrm{Y})=1.00000$

$\mathrm{H} 2 \quad / 1 \quad 6.033766 \quad 1 * 1 * 1 \quad \mathrm{STAT}=\mathrm{Y} \quad \mathrm{PRED}=2.260 \mathrm{RANGE}=2.258 \mathrm{WIDTH}(\mathrm{Y})=2.260 \mathrm{RESP}(\mathrm{Y})=0.5190 \mathrm{HSQC}=\mathrm{C} 2$

$\mathrm{H6} / 1 \quad 6.085811 \quad 1 * 1 * 1 \quad \mathrm{STAT}=\mathrm{Y} \quad \mathrm{PRED}=3.204 \mathrm{RANGE}=3.204 \mathrm{WIDTH}(\mathrm{Y})=2.300 \mathrm{RESP}(\mathrm{Y})=0.6540 \mathrm{HSQC}=\mathrm{C} 6$

H7 $/ 1 \quad 4.438954 \quad 1 * 1 * 1 \quad$ STAT $=Y \quad P R E D=2.409$ RANGE $=2.408$ WIDTH $(Y)=2.409 \operatorname{RESP}(\mathrm{Y})=0.5040 \mathrm{HSQC}=\mathrm{C} 7$

$\mathrm{H} 8 \quad / 1 \quad 4.102838 \quad 1 * 1 * 1 \quad \mathrm{STAT}=\mathrm{Y} \quad \mathrm{PRED}=2.974 \mathrm{RANGE}=2.974 \mathrm{WIDTH}(\mathrm{Y})=2.400 \mathrm{RESP}(\mathrm{Y})=0.5800 \mathrm{HSQC}=\mathrm{C} 8$

$\mathrm{H} 12 / 1 \quad 7.028229 \quad 1 * 1 * 1 \quad \mathrm{STAT}=\mathrm{Y} \quad \mathrm{PRED}=1.952 \mathrm{RANGE}=1.948 \mathrm{WIDTH}(\mathrm{Y})=1.952 \operatorname{RESP}(\mathrm{Y})=0.4920 \mathrm{HSQC}=\mathrm{C} 12$

$\mathrm{H} 13 / 1 \quad 6.811617 \quad 1 * 1 * 1 \quad \mathrm{STAT}=\mathrm{Y} \quad \mathrm{PRED}=3.200 \mathrm{RANGE}=3.197 \mathrm{WIDTH}(\mathrm{Y})=3.200 \mathrm{RESP}(\mathrm{Y})=1.0000 \mathrm{HSQC}=\mathrm{C} 13$

$\mathrm{H} 16 / 1 \quad 7.156230 \quad 1 * 1 * 1 \quad \mathrm{STAT}=\mathrm{Y} \quad \mathrm{PRED}=2.097 \mathrm{RANGE}=2.097 \mathrm{WIDTH}(\mathrm{Y})=2.097 \operatorname{RESP}(\mathrm{Y})=0.6850 \mathrm{HSQC}=\mathrm{C} 16$

$\mathrm{H} 26 / 1 \quad 6.059022 \quad 1 * 1 * 1 \quad \mathrm{STAT}=\mathrm{Y} \quad \mathrm{PRED}=2.642 \mathrm{RANGE}=2.638 \mathrm{WIDTH}(\mathrm{Y})=2.642 \operatorname{RESP}(\mathrm{Y})=0.7110 \mathrm{HSQC}=\mathrm{C} 26$

$\mathrm{H} 29 / 1 \quad 5.337107 \quad 1 * 1 * 1 \quad \mathrm{STAT}=\mathrm{Y} \quad \mathrm{PRED}=2.095 \mathrm{RANGE}=2.095 \mathrm{WIDTH}(\mathrm{Y})=2.095 \mathrm{RESP}(\mathrm{Y})=0.4580 \mathrm{HSOC}=\mathrm{C} 29$

$\mathrm{H} 30 / 1 \quad 3.966907 \quad 1 * 1 * 1 \quad \mathrm{STAT}=\mathrm{Y} \quad \mathrm{PRED}=2.456 \mathrm{RANGE}=2.456 \mathrm{WIDTH}(\mathrm{Y})=2.900 \operatorname{RESP}(\mathrm{Y})=0.4930 \mathrm{HSQC}=\mathrm{C} 30$

$\mathrm{H} 31 / 1 \quad 4.712497 \quad 1 * 1 * 1 \quad \mathrm{STAT}=\mathrm{Y} \quad \mathrm{PRED}=3.329 \mathrm{RANGE}=3.327 \mathrm{WIDTH}(\mathrm{Y})=3.329 \operatorname{RESP}(\mathrm{Y})=0.6280 \mathrm{HSQC}=\mathrm{C} 31$

$\mathrm{H} 33 / 1 \quad 7.122883 \quad 1 * 1 * 1 \quad \mathrm{STAT}=\mathrm{Y} \quad \mathrm{PRED}=2.622 \mathrm{RANGE}=2.618 \mathrm{WIDTH}(\mathrm{Y})=2.622 \operatorname{RESP}(\mathrm{Y})=0.5930 \mathrm{HSQC}=\mathrm{C} 33$

$\mathrm{H} 36 / 1 \quad 6.773527 \quad 1 * 1 * 1 \quad \mathrm{STAT}=\mathrm{Y} \quad \mathrm{PRED}=2.516 \mathrm{RANGE}=2.516 \mathrm{WIDTH}(\mathrm{Y})=2.516 \mathrm{RESP}(\mathrm{Y})=0.5170 \mathrm{HSQC}=\mathrm{C} 36$

$\mathrm{H} 37 / 1 \quad 6.934001 \quad 1 * 1 * 1 \quad \mathrm{STAT}=\mathrm{Y} \quad \mathrm{PRED}=1.952 \mathrm{RANGE}=1.948 \mathrm{WIDTH}(\mathrm{Y})=1.952 \mathrm{RESP}(\mathrm{Y})=0.7080 \mathrm{HSQC}=\mathrm{C} 37$

$\mathrm{H} 42 / 1 \quad 6.036050 \quad 1 * 1 * 1 \quad \mathrm{STAT}=\mathrm{Y} \quad \mathrm{PRED}=2.330 \mathrm{RANGE}=2.328 \mathrm{WIDTH}(\mathrm{Y})=2.330 \mathrm{RESP}(\mathrm{Y})=0.5260 \mathrm{HSQC}=\mathrm{C} 42$

$\mathrm{H} 50 / 1 \quad 4.112117 \quad 1 * 1 * 1 \quad \mathrm{STAT}=\mathrm{Y} \quad \mathrm{PRED}=6.253 \mathrm{RANGE}=6.253 \mathrm{WIDTH}(\mathrm{Y})=3.100 \mathrm{RESP}(\mathrm{Y})=0.8200 \mathrm{HSQC}=\mathrm{C} 50$

$\mathrm{H} 51 / 1 \quad 4.199945 \quad 1 * 1 * 1 \quad \mathrm{STAT}=\mathrm{Y} \quad \mathrm{PRED}=2.962 \mathrm{RANGE}=2.958 \mathrm{WIDTH}(\mathrm{Y})=2.962 \mathrm{RESP}(\mathrm{Y})=0.5450 \mathrm{HSQC}=\mathrm{C} 51$

$\mathrm{H} 53 / 1 \quad 7.130297 \quad 1 * 1 * 1 \quad \mathrm{STAT}=\mathrm{Y} \quad \mathrm{PRED}=1.993 \mathrm{RANGE}=1.988 \mathrm{WIDTH}(\mathrm{Y})=1.993 \mathrm{RESP}(\mathrm{Y})=0.7620 \mathrm{HSQC}=\mathrm{C} 53$

$\mathrm{H} 56 / 1 \quad 6.809154 \quad 1 * 1 * 1 \quad \mathrm{STAT}=\mathrm{Y} \quad \mathrm{PRED}=2.600 \mathrm{RANGE}=2.598 \mathrm{WIDTH}(\mathrm{Y})=2.600 \mathrm{RESP}(\mathrm{Y})=0.9260 \mathrm{HSQC}=\mathrm{C} 56$

$\mathrm{H} 57 / 1 \quad 7.022053 \quad 1 * 1 * 1 \quad \mathrm{STAT}=\mathrm{Y} \quad \mathrm{PRED}=1.441 \mathrm{RANGE}=1.439 \mathrm{WIDTH}(\mathrm{Y})=1.441 \mathrm{RESP}(\mathrm{Y})=0.7640 \mathrm{HSQC}=\mathrm{C} 57$

$\mathrm{H} 68 / 1 \quad 6.114142 \quad 1 * 1 * 1 \quad \mathrm{STAT}=\mathrm{Y} \quad \mathrm{PRED}=2.528 \mathrm{RANGE}=2.528 \mathrm{WIDTH}(\mathrm{Y})=2.528 \mathrm{RESP}(\mathrm{Y})=0.5210 \mathrm{HSQC}=\mathrm{C} 68$

$\mathrm{H} 71 / 1 \quad 4.735766 \quad 1 * 1 * 1 \quad \mathrm{STAT}=\mathrm{Y} \quad \mathrm{PRED}=3.622 \mathrm{RANGE}=3.617 \mathrm{WIDTH}(\mathrm{Y})=3.622 \mathrm{RESP}(\mathrm{Y})=0.6520 \mathrm{HSQC}=\mathrm{C} 71$

$\mathrm{H} 72 / 1 \quad 4.160493 \quad 1 * 1 * 1 \quad$ STAT $=\mathrm{Y} \quad \mathrm{PRED}=7.715 \mathrm{RANGE}=7.715 \mathrm{WIDTH}(\mathrm{Y})=3.800 \mathrm{RESP}(\mathrm{Y})=0.7820 \mathrm{HSQC}=\mathrm{C} 72$

$\mathrm{H} 73 \mathrm{~A} / 1 \quad 3.020091 \quad 1 * 1 * 1 \quad \mathrm{STAT}=\mathrm{Y} \quad \mathrm{PRED}=3.664 \mathrm{RANGE}=3.664 \mathrm{WIDTH}(\mathrm{Y})=3.664 \mathrm{RESP}(\mathrm{Y})=0.5510 \mathrm{HSQC}=\mathrm{C} 73$

$\mathrm{H} 73 \mathrm{~B} / 1 \quad 2.569685 \quad 1 * 1 * 1 \quad \mathrm{STAT}=\mathrm{Y} \quad \mathrm{PRED}=3.694 \mathrm{RANGE}=3.694 \mathrm{WIDTH}(\mathrm{Y})=3.694 \mathrm{RESP}(\mathrm{Y})=0.7250 \mathrm{HSQC}=\mathrm{C} 73$

$\mathrm{H} 75 / 1 \quad 6.995720 \quad 1 * 1 * 1 \quad \mathrm{STAT}=\mathrm{Y} \quad \mathrm{PRED}=3.034 \mathrm{RANGE}=3.034 \mathrm{WIDTH}(\mathrm{Y})=3.034 \mathrm{RESP}(\mathrm{Y})=0.5360 \mathrm{HSQC}=\mathrm{C} 75$

$\mathrm{H} 78 / 1 \quad 6.866427 \quad 1 * 1 * 1 \quad \mathrm{STAT}=\mathrm{Y} \quad \mathrm{PRED}=2.396 \mathrm{RANGE}=2.396 \mathrm{WIDTH}(\mathrm{Y})=2.396 \mathrm{RESP}(\mathrm{Y})=0.4860 \mathrm{HSQC}=\mathrm{C} 78$

$\mathrm{H} 79 / 1 \quad 6.926717 \quad 1 * 1 * 1 \quad \mathrm{STAT}=\mathrm{Y} \quad \mathrm{PRED}=2.751 \mathrm{RANGE}=2.748 \mathrm{WIDTH}(\mathrm{Y})=2.751 \operatorname{RESP}(\mathrm{Y})=0.5180 \mathrm{HSQC}=\mathrm{C} 79$

COUPLING CONSTANTS(HZ):

\begin{tabular}{|c|c|c|c|c|c|}
\hline J85_86 & 2.3173 & $\mathrm{~J} \mathrm{H} 2$ & H6 & $\mathrm{STAT}=\mathrm{Y}$ & $\mathrm{PRED}=2.230 \mathrm{RANGE}=0.890$ \\
\hline J87_88 & 3.2614 & J H7 & H8 & $\mathrm{STAT}=\mathrm{Y}$ & $\mathrm{PRED}=3.680 \mathrm{RANGE}=2.800$ \\
\hline J89_90 & 8.3488 & J H12 & H13 & $\mathrm{STAT}=\mathrm{Y}$ & $\mathrm{PRED}=8.260 \mathrm{RANGE}=0.500$ \\
\hline 91 & 679 & $\mathrm{H} 12$ & H16 & $\mathrm{ATT}=\mathrm{Y}$ & $\mathrm{PRED}=2.030 \mathrm{RANGE}=0.800$ \\
\hline $0-91$ & 010 & H13 & H16 & $\mathrm{AT}=\mathrm{Y}$ & $\mathrm{PRED}=0.430 \mathrm{RANGE}=0.320$ \\
\hline $88^{-} 99$ & 855 & J H29 & $\mathrm{H} 30$ & $\mathrm{STAT}=\mathrm{Y}$ & $\mathrm{PRED}=1.130 \mathrm{RANGE}=2.200$ \\
\hline J99_100 & 1.9076 & J H30 & H31 & STAT $=Y$ & $\mathrm{PRED}=1.540 \mathrm{RANGE}=2.200$ \\
\hline J101_102 & 0.1020 & J H33 & H36 & $\mathrm{STAT}=\mathrm{Y}$ & $\mathrm{PRED}=0.430 \mathrm{RANGE}=0.320$ \\
\hline 101_103 & & J H33 & H37 & $\mathrm{STAT}=\mathrm{Y}$ & $\mathrm{PRED}=2.030 \mathrm{RANGE}=0.800$ \\
\hline $102 \_103$ & 2418 & J H36 & H37 & $\mathrm{STAT}=\mathrm{Y}$ & $\mathrm{PRED}=\varepsilon$ \\
\hline $108 \_109$ & 4825 & J H50 & H51 & $\mathrm{STAT}=\mathrm{Y}$ & $\mathrm{PRED}=3$ \\
\hline $110 \_111$ & 8372 & J H53 & H56 & $\mathrm{STAT}=\mathrm{Y}$ & $\mathrm{PRED}=0.430 \mathrm{RANGE}=0.320$ \\
\hline 110_112 & 2048 & J H53 & H57 & STAT $=Y$ & $\mathrm{PRED}=2.030 \mathrm{RANGE}=0.800$ \\
\hline J111_112 & 3514 & J H56 & H57 & $\mathrm{STAT}=\mathrm{Y}$ & $\mathrm{PRED}=8.260 \mathrm{RA}$ \\
\hline J120_121 & 8.3597 & J H71 & $\mathrm{H} 72$ & $\mathrm{STAT}=\mathrm{Y}$ & $\mathrm{PRED}=10.380 \mathrm{RANGE}=2.000$ \\
\hline J121_122 & & J H72 & H73A & $\mathrm{STAT}=\mathrm{Y}$ & $\mathrm{PRED}=6.680 \mathrm{RANGE}=2.800$ \\
\hline 121_123 & & J H72 & H73B & $\mathrm{STAT}=\mathrm{Y}$ & PRED $=9.720$ RAN \\
\hline $122 \_123$ & -16.29 & J H73A & H73B & $\mathrm{STAT}=\mathrm{Y}$ & $\mathrm{PRED}=-14.780 \mathrm{RANGE}=1.280$ \\
\hline $24 \_125$ & & J H75 & $\mathrm{H} 78$ & STAT $=Y$ & $\mathrm{PRED}=0.430 \mathrm{RANGE}=0.320$ \\
\hline J124_126 & & J H75 & H79 & $\mathrm{STAT}=\mathrm{Y}$ & $\mathrm{PRED}=2.030 \mathrm{RANGE}=0.800$ \\
\hline $\mathrm{J} 125-126$ & 7.9968 & J H78 & H79 & $\mathrm{STAT}=\mathrm{Y}$ & $\mathrm{PRED}=8.260 \mathrm{RANGE}=0.500$ \\
\hline
\end{tabular}

CONTROL PARAMETERS:

Solvent $=$ none $($ def. $99 \%$ enriched $)$

$1.000=$ Concentration $($ vol $\%$, def $=1.0 \%)$

$0.00100000=$ Minimum line-intensity

$0.00100000=$ Diagonalization criterium (not in use)

$899.93600000=$ FIELD $(1 \mathrm{H}, \mathrm{MHz})$, used to transform shifts to ppms

$19.93616218=$ Left frequency $(\mathrm{ppm})$

$-10.17736898=$ Right frequency $(\mathrm{ppm})$

$10.000=$ Acquisition time (s, for QMTLS)

$0.000=$ Line-width (for modes D, P \& T, $0=$ use defaults)

$0.206760077=$ Data-point resolution $(\mathrm{Hz})$

$-8.490=$ GAUSSIAN $(\%, 0=$ use default from INF $)$

$-0.342=$ Dispersion contribution $(\%, 0=$ use default from INF)

$0.00000000=$ Decoupling frequency (for DORES) 
S20. The ${ }^{1} \mathrm{H}$ NMR fingerprint of $\mathbf{2}$ (PERCH .pms file format).

CHEMICAL SHIFTS(PPM):

\begin{tabular}{|c|c|c|c|c|}
\hline OTON & $2 * \mathrm{SPIN}=1$ & & & POPULATION(Y)= 1.00000 \\
\hline $\mathrm{H} 2 / 1$ & 5.989007 & $1 * 1 * 1$ & $\mathrm{STAT}=\mathrm{N}$ & $\mathrm{PRED}=5.965 \mathrm{RANGE}=0.229 \mathrm{WIDTH}(\mathrm{Y})=2.760 \mathrm{RESP}(\mathrm{Y})=0.5414 \mathrm{HSQC}=\mathrm{C} 2$ \\
\hline$/ 1$ & & $1 * 1$ & $\mathrm{AT}=\mathrm{N}$ & $\mathrm{ED}=6.143 \mathrm{RANGE}=0.218 \mathrm{WIDTH}(\mathrm{Y})=3.163 \mathrm{RESP}(\mathrm{Y})=0.5948 \mathrm{HSQC}=\mathrm{C} 6$ \\
\hline$/ 1$ & & $1 * 1 * 1$ & & $378 \mathrm{R} A \mathrm{NGF}=0516 \mathrm{WHDTH}(\mathrm{Y})=2071 \mathrm{RESP}(\mathrm{Y})$ \\
\hline & & $1 * 1 * 1$ & & DID - \\
\hline $\mathrm{H} 12 / 1$ & & $1 * 1 * 1$ & $\mathrm{AT}=\mathrm{N}$ & $\operatorname{PRED}=7.378 \mathrm{RANGE}=0.307 \mathrm{WIDTH}(\mathrm{Y})=3.002 \operatorname{RESP}(\mathrm{Y})=0.6727 \mathrm{HSQC}=\mathrm{C} 12$ \\
\hline $\mathrm{H} 13 / 1$ & & $1 * 1 * 1$ & $\mathrm{ATT}=\mathrm{N}$ & $\mathrm{PRED}=6.795 \mathrm{RANGE}=0.257 \mathrm{WIDTH}(\mathrm{Y})=3.941 \operatorname{RESP}(\mathrm{Y})=1.0000 \mathrm{HSQC}=\mathrm{C} 13$ \\
\hline $\mathrm{H} 16 / 1$ & & $1 * 1 * 1$ & $\mathrm{STAT}=\mathrm{N}$ & $\mathrm{PRED}=6.795 \mathrm{RANGE}=0.618 \mathrm{WIDTH}(\mathrm{Y})=2.697 \mathrm{RESP}(\mathrm{Y})=0.5787 \mathrm{HSQC}=\mathrm{C} 16$ \\
\hline $9 / 1$ & & $1 * 1 * 1$ & $\Gamma A T=\mathrm{N}$ & $\mathrm{PRED}=5.283 \mathrm{RANGE}=0.359 \mathrm{WIDTH}(\mathrm{Y})=$ \\
\hline$/ 1$ & & $1 * 1 * 1$ & $=\mathrm{N}$ & $\mathrm{PRED}=$ \\
\hline & & $1 * 1 * 1$ & $=\mathrm{N}$ & $\mathrm{C} 31$ \\
\hline & & $1 *$ & & $=\mathrm{C} 33$ \\
\hline & & & & $=\mathrm{C} 36$ \\
\hline & & $1 * 1 * 1$ & & $\mathrm{C}=\mathrm{C} 37$ \\
\hline & & & & $\mathrm{C}=\mathrm{C} 44$ \\
\hline & & & & C48 \\
\hline & & $1 * 1 * 1$ & & C49 \\
\hline & & $1 * 1 * 1$ & & $\mathrm{C} 50$ \\
\hline & & & & C51 \\
\hline & & $1 * 1 * 1$ & $\mathrm{STAT}=\mathrm{N}$ & $\mathrm{C}=\mathrm{C} 54$ \\
\hline & & $1 * 1 * 1$ & & $\mathrm{C}=\mathrm{C} 55$ \\
\hline & & & & $=\mathrm{C} 58$ \\
\hline & & & & \\
\hline & & & & $\mathrm{C}=\mathrm{C} 70$ \\
\hline & & & $\mathrm{AT}=\mathrm{N}$ & $\mathrm{C}=\mathrm{C} 70$ \\
\hline & & $1 * 1 * 1$ & $\mathrm{AT}=\mathrm{N}$ & $3 \mathrm{RANGE}=0.390 \mathrm{WIDTH}(\mathrm{Y})=3.1$ \\
\hline $\mathrm{H} 72 / 1$ & & $1 * 1 * 1$ & $\mathrm{STAT}=\mathrm{N}$ & $\mathrm{PRED}=4.881 \mathrm{RANGE}=0.370 \mathrm{WIDTH}(\mathrm{Y})=3.139 \mathrm{RESP}(\mathrm{Y})=0.6995 \mathrm{HSQC}=\mathrm{C} 72$ \\
\hline $\mathrm{H} 75 / 1$ & & $1 * 1 * 1$ & $\mathrm{STAT}=\mathrm{N}$ & $\operatorname{PRED}=7.070 \mathrm{RANGE}=0.496 \mathrm{WIDTH}(\mathrm{Y})=3.393 \operatorname{RESP}(\mathrm{Y})=0.6922 \mathrm{HSQC}=\mathrm{C} 75$ \\
\hline & & $1 * 1 * 1$ & $\mathrm{STAT}=\mathrm{N}$ & $\mathrm{PRED}=6.774 \mathrm{RANGE}=0.270 \mathrm{WIDTH}(\mathrm{Y})=3.107 \operatorname{RESP}(\mathrm{Y})=0.7159 \mathrm{HSQC}=\mathrm{C} 76$ \\
\hline & & $1 * 1 * 1$ & $\mathrm{TAT}=\mathrm{N}$ & $\operatorname{PRED}=7.031 \mathrm{RANGE}=0.390 \mathrm{WIDTH}(\mathrm{Y})=4.706 \operatorname{RESP}(\mathrm{Y})=0.9747 \mathrm{HSQC}=\mathrm{C} 79$ \\
\hline
\end{tabular}

COUPLING CONSTANTS(HZ):

\begin{tabular}{|c|c|c|c|c|c|}
\hline 85_86 & 2.3888 & J H2 & H6 & $\mathrm{STAT}=\mathrm{N}$ & $\mathrm{PRED}=2.185 \mathrm{RANGE}=1.000$ \\
\hline $77_{-8}^{-8}$ & 3.5741 & J H7 & $\mathrm{H} 8$ & $\mathrm{STAT}=\mathrm{N}$ & $\mathrm{PRED}=3.378 \mathrm{RANGE}=2.000$ \\
\hline $89-90$ & 802 & J H12 & H13 & STAT $=\mathrm{N}$ & $\mathrm{EDD}=8.401 \mathrm{RANGE}=1.000$ \\
\hline 991 & 1622 & J H12 & H16 & STAT $=\mathrm{N}$ & $\mathrm{PRED}=2.010 \mathrm{RANGE}=1.000$ \\
\hline 0 & 0052 & J H13 & H16 & $\mathrm{STAT}=\mathrm{N}$ & $\mathrm{PRED}=0.514 \mathrm{RANGE}=1.000$ \\
\hline J97_98 & 0178 & J H29 & $\mathrm{H} 30$ & $\mathrm{STAT}=\mathrm{N}$ & $\mathrm{PRED}=2.714 \mathrm{RANGE}=2.000$ \\
\hline J98_99 & 8314 & J H30 & H31 & $\mathrm{STAT}=\mathrm{Y}$ & $\mathrm{PRED}=2.813 \mathrm{RANGE}=2.040$ \\
\hline 100_101 & 0254 & J H33 & H36 & $\mathrm{STAT}=\mathrm{N}$ & $0 \mathrm{RANGE}=1.000$ \\
\hline 100_102 & 762 & J H33 & H37 & $\mathrm{STAT}=\mathrm{N}$ & PRED $=$ \\
\hline $101^{-} 102$ & 4254 & J H36 & H37 & STAT $=\mathrm{N}$ & $\mathrm{PRED}=8.1$ \\
\hline 7 7_108 & 4263 & J H44 & $\mathrm{H} 48$ & $\mathrm{STAT}=\mathrm{N}$ & $\mathrm{PRED}=2.184 \mathrm{RANGE}=1.000$ \\
\hline $99-110$ & & J H49 & H50 & & $\mathrm{PRED}=1$ \\
\hline J110_111 & & J H50 & H51 & $\mathrm{T}=\mathrm{N}$ & $\mathrm{GE}=2.000$ \\
\hline $\mathrm{J} 112$ & & $\mathrm{H} 54$ & H55 & & PRED $=$ \\
\hline $12 \_114$ & & 54 & H58 & $\mathrm{AT}=\mathrm{N}$ & $\mathrm{PRED}=2.0$ \\
\hline 13_-114 & 0943 & J H55 & H58 & $\mathrm{STAT}=\mathrm{N}$ & $\mathrm{PRED}=0$ \\
\hline $121 \_122$ & 18.4766 & $\mathrm{~J} \mathrm{H} 70 \mathrm{~A}$ & $\mathrm{H} 70 \mathrm{~B}$ & $\mathrm{STAT}=\mathrm{N}$ & PRED $=-15.784$ RANGE $=1.34$ \\
\hline J121_123 & 9900 & $\mathrm{~J} \mathrm{H} 70$ & H71 & $\mathrm{STAT}=\mathrm{N}$ & $\mathrm{PRED}=4.514 \mathrm{RANGE}=2.000$ \\
\hline $121 \_124$ & 0009 & $\mathrm{~J} \mathrm{H} 7 \mathrm{C}$ & $\mathrm{H} 72$ & $\mathrm{STAT}=\mathrm{N}$ & $\mathrm{PRED}=2.520 \mathrm{RANGE}=1.000$ \\
\hline J122_123 & 5059 & $\mathrm{~J} \mathrm{H} 70 \mathrm{~B}$ & $\mathrm{H} 71$ & $\mathrm{STAT}=\mathrm{N}$ & $\mathrm{PRED}=11.737 \mathrm{RANGE}=2.000$ \\
\hline J123_124 & 4084 & J H71 & $\mathrm{H} 72$ & $\mathrm{STAT}=\mathrm{N}$ & $\mathrm{PRED}=1.146 \mathrm{RANGE}=2.000$ \\
\hline & & J H75 & H76 & $\mathrm{STAT}=\mathrm{N}$ & $\mathrm{PRED}=8.185 \mathrm{RANGE}=1.000$ \\
\hline $25 \_127$ & & & $\mathrm{H} 79$ & $\mathrm{STAT}=\mathrm{N}$ & $\mathrm{PRED}=2.083 \mathrm{RANGE}=1.000$ \\
\hline $26-127$ & 0.0084 & J H76 & H79 & $\mathrm{STAT}=\mathrm{N}$ & $\mathrm{PRED}=0.500 \mathrm{RANGE}=1.000$ \\
\hline
\end{tabular}

CONTROL PARAMETERS:

Solvent $=$ none $($ def. $99 \%$ enriched $)$

$1.000=$ Concentration $($ vol $\%, \mathrm{def}=1.0 \%)$

$0.00100000=$ Minimum line-intensity

$0.00100000=$ Diagonalization criterium (not in use)

$900.07850000=\operatorname{FIELD}(1 \mathrm{H}, \mathrm{MHz})$, used to transform shifts to ppms

$12.95939808=$ Left frequency $(\mathrm{ppm})$

$-3.00332745=$ Right frequency $(\mathrm{ppm})$

$10.000=$ Acquisition time $(\mathrm{s}$, for QMTLS $)$

$0.000=$ Line-width (for modes D, P \& T, $0=$ use defaults)

$0.129250004=$ Data-point resolution $(\mathrm{Hz})$

$-4.670=$ GAUSSIAN $(\%, 0=$ use default from INF $)$

$5.139=$ Dispersion contribution $(\%, 0=$ use default from INF)

$0.00000000=$ Decoupling frequency (for DORES) 
S21. The ${ }^{1} \mathrm{H}$ NMR fingerprint of major conformer of $\mathbf{3}$ (PERCH .pms file format).

\begin{tabular}{|c|c|c|c|c|}
\hline \multicolumn{5}{|c|}{ СНЕIVIL AL SНIF I (РPIM): } \\
\hline PROTON & & SPECIE & $\mathrm{S}=1 \mathrm{H}$ & POPULATION $(\mathrm{Y})=1.00000$ \\
\hline $\mathrm{H} 2 \quad / 1$ & 5.965076 & $1 * 1 * 1$ & $\mathrm{STAT}=\mathrm{Y}$ & $\mathrm{PRED}=5.979 \mathrm{RANGE}=0.169 \mathrm{WIDTH}(\mathrm{Y})=1.270 \mathrm{RESP}(\mathrm{Y})=1.0000 \mathrm{HSQC}=\mathrm{C} 2$ \\
\hline H6 & 013776 & $1 * 1 * 1$ & $\mathrm{STAT}=\mathrm{Y}$ & $\mathrm{PRED}=6.073 \mathrm{RANGE}=0.209 \mathrm{WIDTH}(\mathrm{Y})=1.363 \operatorname{RESP}(\mathrm{Y})=1.0000 \mathrm{HSQC}=\mathrm{C} 6$ \\
\hline & 147872 & $1 * 1 * 1$ & $\mathrm{STAT}=\mathrm{Y}$ & $\mathrm{PRED}=3.489 \mathrm{RANGE}=0.999 \mathrm{WIDTH}(\mathrm{Y})=1.699 \operatorname{RESP}(\mathrm{Y})=1.0000 \mathrm{HSQC}=\mathrm{C} 7$ \\
\hline H8 & 281835 & $1 * 1 * 1$ & $\mathrm{STAT}=\mathrm{Y}$ & $\mathrm{PRED}=3.267 \mathrm{RANGE}=0.519 \mathrm{WIDTH}(\mathrm{Y})=1.350 \mathrm{RESP}(\mathrm{Y})=1.0000 \mathrm{HSQC}=\mathrm{C} 8$ \\
\hline $\mathrm{H} 12 / \mathrm{l}$ & 6.848257 & $1 * 1 * 1$ & $\mathrm{STAT}=\mathrm{Y}$ & $\mathrm{PRED}=7.238 \mathrm{RANGE}=0.209 \mathrm{WIDTH}(\mathrm{Y})=1.264 \operatorname{RESP}(\mathrm{Y})=1.0000 \mathrm{HSQC}=\mathrm{C} 12$ \\
\hline $\mathrm{H} 13 / \mathrm{l}$ & & $1 * 1 * 1$ & $\mathrm{STAT}=\mathrm{Y}$ & $\mathrm{PRED}=6.798 \mathrm{RANGE}=0.189 \mathrm{WIDTH}(\mathrm{Y})=1.514 \operatorname{RESP}(\mathrm{Y})=1.0000 \mathrm{HSQC}=\mathrm{C} 13$ \\
\hline $\mathrm{H} 16 / 1$ & & $1 * 1 * 1$ & $\mathrm{STAT}=\mathrm{Y}$ & $\mathrm{PRED}=6.872 \mathrm{RANGE}=0.386 \mathrm{WIDTH}(\mathrm{Y})=1$ \\
\hline $\mathrm{H} 26 / 1$ & & $1 * 1 * 1$ & $\mathrm{STAT}=\mathrm{Y}$ & $1 \mathrm{RANGE}=0$ \\
\hline $\mathrm{H} 29 / 1$ & & $1 * 1 * 1$ & $\mathrm{STAT}=\mathrm{Y}$ & $36 \mathrm{RANGE}=0$ \\
\hline $\mathrm{H} 30 / \mathrm{l}$ & 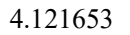 & $1 * 1 * 1$ & $\mathrm{STAT}=\mathrm{Y}$ & $\mathrm{PRED}=4.173 \mathrm{RANGE}=0.556 \mathrm{WIDTH}(\mathrm{Y})=$ \\
\hline $\mathrm{H} 31 / 1$ & & $1 * 1 * 1$ & & $\operatorname{PRED}=2.842 \operatorname{RANGE}=1.289 \operatorname{WIDTH}(\mathrm{Y})=1.750 \operatorname{RESP}(\mathrm{Y})=1.0000$ \\
\hline $\mathrm{H} 33 / 1$ & 7.3 & $1 * 1 * 1$ & $\mathrm{STAT}=\mathrm{Y}$ & $\mathrm{PRED}=6.776 \mathrm{RANGE}=0.279 \mathrm{WIDTH}(\mathrm{Y})=1.654 \mathrm{RESP}(\mathrm{Y})=1.0000$ \\
\hline $\mathrm{H} 36 / 1$ & & $1 * 1 * 1$ & $\mathrm{STAT}=\mathrm{Y}$ & $\operatorname{PRED}=6.726 \mathrm{RANGE}=0.159 \operatorname{WIDTH}(\mathrm{Y})=1.425 \operatorname{RESP}(\mathrm{Y})=$ \\
\hline $\mathrm{H} 37 / \mathrm{l}$ & & $1 * 1 * 1$ & $\mathrm{ST} A$ & $=\mathrm{C} 37$ \\
\hline $\mathrm{H} 46 / \mathrm{l}$ & & $1 * 1 * 1$ & STA & PRED \\
\hline $\mathrm{H} 49 / 1$ & & $1 * 1 * 1$ & $\mathrm{STAT}=\mathrm{Y}$ & $\mathrm{PRED}=4.657 \mathrm{RANGE}=0.339 \mathrm{WIDTH}(\mathrm{Y})=2.782 \operatorname{RESP}(\mathrm{Y})=$ \\
\hline $50 / 1$ & & $1 * 1 * 1$ & $\mathrm{STAT}=\mathrm{Y}$ & $\mathrm{PRED}=3.972 \mathrm{RANGE}=0.289 \mathrm{WIDTH}(\mathrm{Y})=2.667 \mathrm{RESP}(\mathrm{Y})=1.00$ \\
\hline $\mathrm{H} 51 \mathrm{~A} / 1$ & 20488 & $1 * 1 * 1$ & $\mathrm{STAT}=\mathrm{Y}$ & $\mathrm{PRED}=2.696 \mathrm{RANGE}=0.179 \mathrm{WIDTH}(\mathrm{Y})=3.126 \mathrm{RESP}(\mathrm{Y})=1.0000 \mathrm{HSQC}=\mathrm{C}$ \\
\hline $\mathrm{H} 51 \mathrm{~B} / 1$ & 2.846560 & $1 * 1 * 1$ & $\mathrm{STAT}=\mathrm{Y}$ & $\mathrm{PRED}=2.768 \mathrm{RANGE}=0.199 \mathrm{WIDTH}(\mathrm{Y})=2.647 \operatorname{RESP}(\mathrm{Y})=1.0000 \mathrm{HSQC}=\mathrm{C} 5$ \\
\hline $\mathrm{H} 53 / 1$ & & $1 * 1 * 1$ & $\mathrm{STAT}=\mathrm{Y}$ & $\mathrm{PRED}=6.635 \mathrm{RANGE}=0.508 \mathrm{WIDTH}(\mathrm{Y})=1.775 \mathrm{RESP}(\mathrm{Y})=1.0000 \mathrm{HSQC}=\mathrm{C} 53$ \\
\hline $\mathrm{H} 56 / 1$ & & & & \\
\hline $\mathrm{H} 57 / \mathrm{l}$ & 6.728062 & $1 * 1 * 1$ & $\mathrm{STAT}=\mathrm{Y}$ & $\mathrm{RED}=7.072 \mathrm{RANGE}=0.399 \mathrm{WIDTH}(\mathrm{Y})=1.797 \mathrm{RESP}(\mathrm{Y})=1.0000 \mathrm{HSQC}=\mathrm{C} 5$ \\
\hline
\end{tabular}

COUPLING CONSTANTS(HZ):

\begin{tabular}{|c|c|c|c|c|c|}
\hline J64_65 & 2.3393 & J H2 & H6 & $\mathrm{STAT}=\mathrm{Y}$ & $\mathrm{PRED}=2.230 \mathrm{RANGE}=0.890$ \\
\hline J66_67 67 & 3.4327 & J H7 & $\mathrm{H} 8$ & $\mathrm{STAT}=\mathrm{Y}$ & $\mathrm{PRED}=3.670 \mathrm{RANGE}=2.800$ \\
\hline J68_69 & 8.2689 & J H12 & H13 & $\mathrm{STAT}=\mathrm{Y}$ & $\mathrm{PRED}=8.260 \mathrm{RANGE}=0.500$ \\
\hline J68_70 & 2.1735 & J H12 & H16 & $\mathrm{STAT}=\mathrm{Y}$ & $\mathrm{PRED}=2.030 \mathrm{RANGE}=0.800$ \\
\hline J69_70 & 0.0037 & J H13 & H16 & $\mathrm{STAT}=\mathrm{Y}$ & $\mathrm{PRED}=0.430 \mathrm{RANGE}=0.320$ \\
\hline J77_78 & 7847 & J H29 & $\mathrm{H} 30$ & $\mathrm{STAT}=\mathrm{Y}$ & $\mathrm{PRED}=1.060 \mathrm{RANGE}=2.000$ \\
\hline 23 & 1.2100 & J H29 & H31 & $\mathrm{STAT}=\mathrm{Y}$ & \\
\hline J78_79 & 1.9551 & J H30 & $\mathrm{H} 31$ & $\mathrm{STAT}=\mathrm{Y}$ & $\mathrm{PRED}=1.590 \mathrm{RANGE}=2.200$ \\
\hline $\mathrm{J} 80+81$ & 0.0002 & J H33 & H36 & $\mathrm{STAT}=\mathrm{Y}$ & $\mathrm{PRED}=0.430 \mathrm{RANGE}=0.320$ \\
\hline $\mathrm{J} 80+82$ & .0838 & J H33 & H37 & $\mathrm{STAT}=\mathrm{Y}$ & $\mathrm{PRED}=2.030 \mathrm{RANGE}=0.800$ \\
\hline J81_82 & 1893 & J H36 & H37 & $\mathrm{STAT}=\mathrm{Y}$ & $\mathrm{PRED}=8.260 \mathrm{RANGE}=0.500$ \\
\hline J87_88 & 0994 & J H49 & $\mathrm{H} 50$ & $\mathrm{STAT}=\mathrm{Y}$ & $\mathrm{PRED}=1.140 \mathrm{RANGE}=2.200$ \\
\hline J88_89 & 229 & J H50 & H51A & $\mathrm{STAT}=\mathrm{Y}$ & .010 RANGE $=2.200$ \\
\hline J88_90 & 4.8605 & J H50 & H51B & $\mathrm{STAT}=\mathrm{Y}$ & $\mathrm{PRED}=4.410 \mathrm{RANGE}=2.800$ \\
\hline J89_90 & 17.3564 & J H51A & H51B & $\mathrm{STAT}=\mathrm{Y}$ & $\mathrm{PRED}=-14.870 \mathrm{RANGE}=1.28$ \\
\hline J91_92 & 0.0266 & J H53 & H56 & $\mathrm{STAT}=\mathrm{Y}$ & $\mathrm{PRED}=0.430 \mathrm{RANGE}=0.320$ \\
\hline J91_93 & .0260 & J H53 & H57 & $\mathrm{STAT}=\mathrm{Y}$ & $\mathrm{PRED}=2.030 \mathrm{RANGE}=0.800$ \\
\hline J92_93 & 8.1594 & J H56 & H57 & $\mathrm{STAT}=\mathrm{Y}$ & $\mathrm{PRED}=8.260 \mathrm{RANGE}=0.500$ \\
\hline
\end{tabular}

CONTROL PARAMETERS:

Solvent $=$ none $($ def. $99 \%$ enriched $)$

$1.000=$ Concentration $($ vol $\%$, def $=1.0 \%)$

$0.00100000=$ Minimum line-intensity

$0.00100000=$ Diagonalization criterium (not in use)

$899.93600000=$ FIELD $(1 \mathrm{H}, \mathrm{MHz})$, used to transform shifts to ppms

$19.92595746=$ Left frequency $(\mathrm{ppm})$

$-10.18757262=$ Right frequency $(\mathrm{ppm})$

$10.000=$ Acquisition time (s, for QMTLS)

$0.000=$ Line-width (for modes D, P \& T, $0=$ use defaults)

$0.206760153=$ Data-point resolution $(\mathrm{Hz})$

$14.222=$ GAUSSIAN $(\%, 0=$ use default from INF $)$

$-0.167=$ Dispersion contribution $(\%, 0=$ use default from INF)

$0.00000000=$ Decoupling frequency (for DORES) 
S22. The ${ }^{1} \mathrm{H}$ NMR fingerprint of minor conformer of $\mathbf{3}$ (PERCH .pms file format).

\begin{tabular}{|c|c|c|c|c|}
\hline \multicolumn{5}{|c|}{ С HEVIL AL SHIF IS(PPM): } \\
\hline PROTON & & SPECIE & $\mathrm{S}=1 \mathrm{H}$ & POPULATION $(\mathrm{Y})=1.00000$ \\
\hline $\mathrm{H} 2 \quad / 1$ & 6.022830 & $1 * 1 * 1$ & $\mathrm{STAT}=\mathrm{Y}$ & $\mathrm{PRED}=5.979 \mathrm{RANGE}=0.169 \mathrm{WIDTH}(\mathrm{Y})=1.232 \operatorname{RESP}(\mathrm{Y})=1.0000 \mathrm{HSQC}=\mathrm{C} 2$ \\
\hline H6 & 078180 & $1 * 1 * 1$ & $\mathrm{STAT}=\mathrm{Y}$ & $\mathrm{PRED}=6.073 \mathrm{RANGE}=0.209 \mathrm{WIDTH}(\mathrm{Y})=1.362 \operatorname{RESP}(\mathrm{Y})=1.0000 \mathrm{HSQC}=\mathrm{C} 6$ \\
\hline & 53725 & $1 * 1 * 1$ & $\mathrm{STAT}=\mathrm{Y}$ & $\mathrm{PRED}=3.489 \mathrm{RANGE}=0.999 \mathrm{WIDTH}(\mathrm{Y})=1.698 \operatorname{RESP}(\mathrm{Y})=1.0000 \mathrm{HSQC}=\mathrm{C} 7$ \\
\hline H8 & 095342 & $1 * 1 * 1$ & $\mathrm{STAT}=\mathrm{Y}$ & $\mathrm{PRED}=3.267 \mathrm{RANGE}=0.519 \mathrm{WIDTH}(\mathrm{Y})=1.305 \operatorname{RESP}(\mathrm{Y})=1.0000 \mathrm{HSQC}=\mathrm{C} 8$ \\
\hline $\mathrm{H} 12 / 1$ & 7.037961 & $1 * 1 * 1$ & $\mathrm{STAT}=\mathrm{Y}$ & $\mathrm{PRED}=7.238 \mathrm{RANGE}=0.209 \mathrm{WIDTH}(\mathrm{Y})=1.220 \operatorname{RESP}(\mathrm{Y})=1.0000 \mathrm{HSQC}=\mathrm{C} 12$ \\
\hline $\mathrm{H} 13 / \mathrm{l}$ & 6.816180 & $1 * 1 * 1$ & $\mathrm{STAT}=\mathrm{Y}$ & $\mathrm{PRED}=6.798 \mathrm{RANGE}=0.189 \mathrm{WIDTH}(\mathrm{Y})=1.475 \operatorname{RESP}(\mathrm{Y})=1.0000 \mathrm{HSQC}=\mathrm{C} 13$ \\
\hline $\mathrm{H} 16 / 1$ & & $1 * 1 * 1$ & $\mathrm{STAT}=\mathrm{Y}$ & $\mathrm{PRED}=6.872 \mathrm{RANGE}=0.386 \mathrm{WIDTH}(\mathrm{Y})=1$ \\
\hline $\mathrm{H} 26 / 1$ & & $1 * 1 * 1$ & $\mathrm{STAT}=\mathrm{Y}$ & $1 \mathrm{RANGE}=0$ \\
\hline $\mathrm{H} 29 / 1$ & & $1 * 1 * 1$ & $\mathrm{STAT}=\mathrm{Y}$ & $36 \mathrm{RANGE}=0$ \\
\hline $\mathrm{H} 30 / 1$ & 3035 & $1 * 1 * 1$ & $\mathrm{STAT}=\mathrm{Y}$ & $\mathrm{PRED}=4.173 \mathrm{RANGE}=0.556 \mathrm{WIDTH}(\mathrm{Y})=$ \\
\hline $\mathrm{H} 31 / 1$ & & $1 * 1 * 1$ & & $\mathrm{PRED}=2.842 \mathrm{RANGE}=1.289 \mathrm{WIDTH}(\mathrm{Y})=2.974 \operatorname{RESP}(\mathrm{Y})=1.0000$ \\
\hline $\mathrm{H} 33 / 1$ & 7.0 & $1 * 1 * 1$ & $\mathrm{STAT}=\mathrm{Y}$ & $\mathrm{PRED}=6.776 \mathrm{RANGE}=0.279 \mathrm{WIDTH}(\mathrm{Y})=1.608 \mathrm{RESP}(\mathrm{Y})=1.0000$ \\
\hline $\mathrm{H} 36 / 1$ & & $1 * 1 * 1$ & & $\mathrm{PRED}=6.726 \mathrm{RANGE}=0.159 \operatorname{WIDTH}(\mathrm{Y})=1.816 \operatorname{RESP}(\mathrm{Y})=$ \\
\hline $7 / 1$ & & $1 * 1 * 1$ & $\mathrm{ST} A$ & $=\mathrm{C} 37$ \\
\hline $\mathrm{H} 46 / \mathrm{l}$ & & $1 * 1 * 1$ & STA & PRED \\
\hline $\mathrm{H} 49 / 1$ & & $1 * 1 * 1$ & $\mathrm{STAT}=\mathrm{Y}$ & $\mathrm{PRED}=4.657 \mathrm{RANGE}=0.339 \mathrm{WIDTH}(\mathrm{Y})=2.735 \operatorname{RESP}(\mathrm{Y})=$ \\
\hline $50 / 1$ & & $1 * 1 * 1$ & $\mathrm{STAT}=\mathrm{Y}$ & $\mathrm{PRED}=3.972 \mathrm{RANGE}=0.289 \mathrm{WIDTH}(\mathrm{Y})=3.466 \operatorname{RESP}(\mathrm{Y})=1.0$ \\
\hline $\mathrm{H} 51 \mathrm{~A} / 1$ & 4844 & $1 * 1 * 1$ & $\mathrm{STAT}=\mathrm{Y}$ & $\mathrm{PRED}=2.696 \mathrm{RANGE}=0.179 \mathrm{WIDTH}(\mathrm{Y})=3.036 \mathrm{RESP}(\mathrm{Y})=1.0000 \mathrm{HSQC}=\mathrm{C}$ \\
\hline $\mathrm{H} 51 \mathrm{~B} / 1$ & 2.934562 & $1 * 1 * 1$ & $\mathrm{STAT}=\mathrm{Y}$ & $\mathrm{PRED}=2.768 \mathrm{RANGE}=0.199 \mathrm{WIDTH}(\mathrm{Y})=2.498 \operatorname{RESP}(\mathrm{Y})=1.0000 \mathrm{HSQC}=\mathrm{C} 5$ \\
\hline $\mathrm{H} 53 / 1$ & & $1 * 1 * 1$ & $\mathrm{STAT}=\mathrm{Y}$ & $\mathrm{PRED}=6.635 \mathrm{RANGE}=0.508 \mathrm{WIDTH}(\mathrm{Y})=1.623 \operatorname{RESP}(\mathrm{Y})=1.0000 \mathrm{HSQC}=\mathrm{C} 53$ \\
\hline $\mathrm{H} 56 / 1$ & & $1 * 1 * 1$ & & \\
\hline $\mathrm{H} 57 / \mathrm{l}$ & 6.887306 & $1 * 1 * 1$ & $\mathrm{STAT}=\mathrm{Y}$ & $\mathrm{RED}=7.072 \mathrm{RANGE}=0.399 \mathrm{WIDTH}(\mathrm{Y})=1.718 \mathrm{RESP}(\mathrm{Y})=1.0000 \mathrm{HSQC}=\mathrm{C} 5$ \\
\hline
\end{tabular}

COUPLING CONSTANTS(HZ):

\begin{tabular}{|c|c|c|c|c|c|}
\hline J64_65 & 2.3361 & $\mathrm{~J} \mathrm{H} 2$ & H6 & $\mathrm{STAT}=\mathrm{Y}$ & $\mathrm{PRED}=2.230 \mathrm{RANGE}=0.890$ \\
\hline J66 67 & 3.4319 & J H7 & $\mathrm{H} 8$ & $\mathrm{STAT}=\mathrm{Y}$ & $\mathrm{PRED}=3.670 \mathrm{RANGE}=2.800$ \\
\hline J68_69 & 8.2611 & J H12 & H13 & $\mathrm{STAT}=\mathrm{Y}$ & $\mathrm{PRED}=8.260 \mathrm{RANGE}=0.500$ \\
\hline $\mathrm{J} 688_{-}^{-} 70$ & 2.1671 & J H12 & H16 & $\mathrm{STAT}=\mathrm{Y}$ & $\mathrm{PRED}=2.030 \mathrm{RANGE}=0.800$ \\
\hline J69_70 & 0.0166 & J H13 & H16 & $\mathrm{STAT}=\mathrm{Y}$ & $\mathrm{PRED}=0.430 \mathrm{RANGE}=0.320$ \\
\hline J77_78 & 0.5632 & J H29 & $\mathrm{H} 30$ & $\mathrm{STAT}=\mathrm{Y}$ & $\mathrm{PRED}=1.060 \mathrm{RANGE}=2.000$ \\
\hline J78_79 & 1.9340 & J H30 & H31 & $\mathrm{STAT}=\mathrm{Y}$ & $\mathrm{PRED}=1.590 \mathrm{RANGE}=2.200$ \\
\hline J80_81 & 0.5591 & J H33 & H36 & $\mathrm{STAT}=\mathrm{Y}$ & $\mathrm{PRED}=0.430 \mathrm{RANGE}=0.320$ \\
\hline $\mathrm{J} 80+82$ & 2.2472 & J H33 & H37 & $\mathrm{STAT}=\mathrm{Y}$ & $\mathrm{PRED}=2.030 \mathrm{RANGE}=0.800$ \\
\hline J81_82 & 8.2358 & J H36 & H37 & $\mathrm{STAT}=\mathrm{Y}$ & $\mathrm{PRED}=8.260 \mathrm{RANGE}=0.500$ \\
\hline J87_88 & 0.0022 & J H49 & $\mathrm{H} 50$ & $\mathrm{STAT}=\mathrm{Y}$ & $\mathrm{PRED}=1.140 \mathrm{RANGE}=2.200$ \\
\hline J88_89 & 3.2159 & J H50 & $\mathrm{H} 51 \mathrm{~A}$ & $\mathrm{STAT}=\mathrm{Y}$ & $\mathrm{PRED}=2.010 \mathrm{RANGE}=2.200$ \\
\hline J88_90 & 4.7611 & J H50 & $\mathrm{H} 51 \mathrm{~B}$ & $\mathrm{STAT}=\mathrm{Y}$ & $\mathrm{PRED}=4.410 \mathrm{RANGE}=2.800$ \\
\hline J89_90 & -17.3652 & J H51A & H51B & STAT $=Y$ & $\mathrm{PRED}=-14.870 \mathrm{RANGE}=1.280$ \\
\hline J91_92 & 0.3464 & J H53 & H56 & $\mathrm{STAT}=\mathrm{Y}$ & $\mathrm{PRED}=0.430 \mathrm{RANGE}=0.320$ \\
\hline J91_93 & 2.0125 & J H53 & H57 & $\mathrm{STAT}=\mathrm{Y}$ & $\mathrm{PRED}=2.030 \mathrm{RANGE}=0.800$ \\
\hline J92_93 & 8.1644 & J H56 & H57 & $\mathrm{STAT}=\mathrm{Y}$ & $\mathrm{PRED}=8.260 \mathrm{RANGE}=0.500$ \\
\hline
\end{tabular}

CONTROL PARAMETERS:

Solvent $=$ none $($ def. $99 \%$ enriched $)$

$1.000=$ Concentration $(\mathrm{vol} \%$, def $=1.0 \%)$

$0.00100000=$ Minimum line-intensity

$0.00100000=$ Diagonalization criterium (not in use)

$899.93600000=$ FIELD $(1 \mathrm{H}, \mathrm{MHz})$, used to transform shifts to ppms

$19.92595746=$ Left frequency $(\mathrm{ppm})$

$-10.18757262=$ Right frequency $(\mathrm{ppm})$

$10.000=$ Acquisition time $(\mathrm{s}$, for QMTLS $)$

$0.000=$ Line-width (for modes D, P \& T, $0=$ use defaults)

$0.206760153=$ Data-point resolution $(\mathrm{Hz})$

$60.116=$ GAUSSIAN $(\%, 0=$ use default from INF $)$

$2.109=$ Dispersion contribution $(\%, 0=$ use default from INF)

$0.00000000=$ Decoupling frequency (for DORES) 
S23. The ${ }^{1} \mathrm{H}$ NMR fingerprint of the mixture of $\mathbf{3}$ (PERCH .pms file format).

CHEMICAL SHIFTS(PPM):

CTB1major $\quad 2 *$ SPIN $=1$ SPECIES $=1 \mathrm{H} \quad$ POPULATION $(\mathrm{Y})=0.77669$

$1-\mathrm{H} 2 \quad / 1 \quad 5.965076 \quad 1 * 1 * 1 \quad \mathrm{STAT}=\mathrm{Y} \quad \mathrm{PRED}=5.979 \mathrm{RANGE}=0.169 \mathrm{WIDTH}(\mathrm{Y})=1.270 \mathrm{RESP}(\mathrm{Y})=1.0000 \mathrm{HSQC}=\mathrm{C} 2$

$1-\mathrm{H} 6 \quad / 1 \quad 6.013776 \quad 1 * 1 * 1 \quad$ STAT $=\mathrm{Y} \quad \mathrm{PRED}=6.073$ RANGE $=0.209$ WIDTH$(\mathrm{Y})=1.363 \mathrm{RESP}(\mathrm{Y})=1.0000 \mathrm{HSQC}=\mathrm{C} 6$

$1-\mathrm{H} 7 \quad / 1 \quad 4.147824 \quad 1 * 1 * 1 \quad \mathrm{STAT}=\mathrm{Y} \quad \mathrm{PRED}=3.489 \mathrm{RANGE}=0.999 \mathrm{WIDTH}(\mathrm{Y})=1.733 \mathrm{RESP}(\mathrm{Y})=1.0000 \mathrm{HSQC}=\mathrm{C} 7$

$1-\mathrm{H} 8 \quad / 1 \quad 3.281785 \quad 1 * 1 * 1 \quad$ STAT $=\mathrm{Y} \quad \mathrm{PRED}=3.267 \mathrm{RANGE}=0.519$ WIDTH$(\mathrm{Y})=1.422 \mathrm{RESP}(\mathrm{Y})=1.0000 \mathrm{HSQC}=\mathrm{C} 8$

$1-\mathrm{H} 12 / 1 \quad 6.848206 \quad 1 * 1 * 1 \quad$ STAT $=\mathrm{Y} \quad \mathrm{PRED}=7.238 \mathrm{RANGE}=0.209 \mathrm{WIDTH}(\mathrm{Y})=1.283 \mathrm{RESP}(\mathrm{Y})=1.0000 \mathrm{HSQC}=\mathrm{C} 12$

$1-\mathrm{H} 13 / 1 \quad 6.823857 \quad 1 * 1 * 1 \quad$ STAT $=\mathrm{Y} \quad \mathrm{PRED}=6.798 \mathrm{RANGE}=0.189 \mathrm{WIDTH}(\mathrm{Y})=1.499 \mathrm{RESP}(\mathrm{Y})=1.0000 \mathrm{HSQC}=\mathrm{C} 13$

$1-\mathrm{H} 16 / 1 \quad 7.031613 \quad 1 * 1 * 1 \quad \mathrm{STAT}=\mathrm{Y} \quad \mathrm{PRED}=6.872 \mathrm{RANGE}=0.386 \mathrm{WIDTH}(\mathrm{Y})=1.325 \mathrm{RESP}(\mathrm{Y})=1.0000 \mathrm{HSQC}=\mathrm{C} 16$

$1-\mathrm{H} 26 / 1 \quad 5.800342 \quad 1 * 1 * 1 \quad \mathrm{STAT}=\mathrm{Y} \quad \mathrm{PRED}=5.851 \mathrm{RANGE}=0.366 \mathrm{WIDTH}(\mathrm{Y})=1.452 \mathrm{RESP}(\mathrm{Y})=1.0000 \mathrm{HSQC}=\mathrm{C} 26$

$1-\mathrm{H} 29 / 1 \quad 5.705377 \quad 1 * 1 * 1 \quad$ STAT $=\mathrm{Y} \quad \mathrm{PRED}=5.846 \mathrm{RANGE}=0.379 \mathrm{WIDTH}(\mathrm{Y})=1.842 \operatorname{RESP}(\mathrm{Y})=1.0000 \mathrm{HSQC}=\mathrm{C} 29$

$1-\mathrm{H} 30 / 1 \quad 4.121596 \quad 1 * 1 * 1 \quad \mathrm{STAT}=\mathrm{Y} \quad \mathrm{PRED}=4.173 \mathrm{RANGE}=0.556 \mathrm{WIDTH}(\mathrm{Y})=2.348 \mathrm{RESP}(\mathrm{Y})=1.0000 \mathrm{HSQC}=\mathrm{C} 30$

$1-\mathrm{H} 31 / 1 \quad 4.559281 \quad 1 * 1 * 1 \quad$ STAT $=\mathrm{Y} \quad \mathrm{PRED}=2.842 \mathrm{RANGE}=1.289 \mathrm{WIDTH}(\mathrm{Y})=2.257 \mathrm{RESP}(\mathrm{Y})=1.0000 \mathrm{HSQC}=\mathrm{C} 31$

$1-\mathrm{H} 33 / 1 \quad 7.316613 \quad 1 * 1 * 1 \quad \mathrm{STAT}=\mathrm{Y} \quad \mathrm{PRED}=6.776 \mathrm{RANGE}=0.279 \mathrm{WIDTH}(\mathrm{Y})=1.723 \mathrm{RESP}(\mathrm{Y})=1.0000 \mathrm{HSQC}=\mathrm{C} 33$

$1-\mathrm{H} 36 / 1 \quad 6.842505 \quad 1 * 1 * 1 \quad \mathrm{STAT}=\mathrm{Y} \quad \mathrm{PRED}=6.726 \mathrm{RANGE}=0.159 \mathrm{WIDTH}(\mathrm{Y})=1.444 \operatorname{RESP}(\mathrm{Y})=1.0000 \mathrm{HSQC}=\mathrm{C} 36$

$1-\mathrm{H} 37 / 1 \quad 7.193167 \quad 1 * 1 * 1 \quad$ STAT $=\mathrm{Y} \quad \mathrm{PRED}=7.242 \mathrm{RANGE}=0.549 \mathrm{WIDTH}(\mathrm{Y})=1.956 \mathrm{RESP}(\mathrm{Y})=1.0000 \mathrm{HSQC}=\mathrm{C} 37$

$1-\mathrm{H} 46 / 1 \quad 6.102988 \quad 1 * 1 * 1 \quad \mathrm{STAT}=\mathrm{Y} \quad \mathrm{PRED}=5.966 \mathrm{RANGE}=0.598 \mathrm{WIDTH}(\mathrm{Y})=1.706 \mathrm{RESP}(\mathrm{Y})=1.0000 \mathrm{HSQC}=\mathrm{C} 46$

$1-\mathrm{H} 49 / 1 \quad 4.386622 \quad 1 * 1 * 1 \quad$ STAT $=\mathrm{Y} \quad \mathrm{PRED}=4.657 \mathrm{RANGE}=0.339 \mathrm{WIDTH}(\mathrm{Y})=2.701 \mathrm{RESP}(\mathrm{Y})=1.0000 \mathrm{HSQC}=\mathrm{C} 49$

$1-\mathrm{H} 50 / 1 \quad 3.861219 \quad 1 * 1 * 1 \quad \mathrm{STAT}=\mathrm{Y} \quad \mathrm{PRED}=3.972 \mathrm{RANGE}=0.289 \mathrm{WIDTH}(\mathrm{Y})=2.631 \mathrm{RESP}(\mathrm{Y})=1.0000 \mathrm{HSQC}=\mathrm{C} 50$

$1-\mathrm{H} 51 \mathrm{~A} / 1 \quad 2.820501 \quad 1 * 1 * 1 \quad \mathrm{STAT}=\mathrm{Y} \quad \mathrm{PRED}=2.696 \mathrm{RANGE}=0.179 \mathrm{WIDTH}(\mathrm{Y})=3.114 \mathrm{RESP}(\mathrm{Y})=1.0000 \mathrm{HSQC}=\mathrm{C} 51$

$1-\mathrm{H} 51 \mathrm{~B} / 1 \quad 2.846492 \quad 1 * 1 * 1 \quad \mathrm{STAT}=\mathrm{Y} \quad \mathrm{PRED}=2.768 \mathrm{RANGE}=0.199 \mathrm{WIDTH}(\mathrm{Y})=2.739 \mathrm{RESP}(\mathrm{Y})=1.0000 \mathrm{HSQC}=\mathrm{C} 51$

$1-\mathrm{H} 53 / 1 \quad 6.822558 \quad 1 * 1 * 1 \quad$ STAT $=\mathrm{Y} \quad \mathrm{PRED}=6.635 \mathrm{RANGE}=0.508 \mathrm{WIDTH}(\mathrm{Y})=1.628 \mathrm{RESP}(\mathrm{Y})=1.0000 \mathrm{HSQC}=\mathrm{C} 53$

$1-\mathrm{H} 56 / 1 \quad 6.751769 \quad 1 * 1 * 1 \quad \mathrm{STAT}=\mathrm{Y} \quad \mathrm{PRED}=6.666 \mathrm{RANGE}=0.669 \mathrm{WIDTH}(\mathrm{Y})=1.216 \mathrm{RESP}(\mathrm{Y})=1.0000 \mathrm{HSQC}=\mathrm{C} 56$

$1-\mathrm{H} 57 / 1 \quad 6.728005 \quad 1 * 1 * 1 \quad$ STAT $=\mathrm{Y} \quad \mathrm{PRED}=7.072 \mathrm{RANGE}=0.399 \mathrm{WIDTH}(\mathrm{Y})=1.835 \operatorname{RESP}(\mathrm{Y})=1.0000 \mathrm{HSQC}=\mathrm{C} 57$

CTB1minor $2 *$ SPIN $=1$ SPECIES $=1 \mathrm{H} \quad$ POPULATION $(\mathrm{Y})=0.22331$

$2-\mathrm{H} 2 \quad / 2 \quad 6.022831 \quad 1 * 1 * 1 \quad \mathrm{STAT}=\mathrm{Y} \quad \mathrm{PRED}=5.979 \mathrm{RANGE}=0.169 \mathrm{WIDTH}(\mathrm{Y})=1.305 \mathrm{RESP}(\mathrm{Y})=1.0000 \mathrm{HSQC}=\mathrm{C} 2$

2-H6 $/ 2 \quad 6.078179 \quad 1 * 1 * 1 \quad$ STAT $=\mathrm{Y} \quad \mathrm{PRED}=6.073 \mathrm{RANGE}=0.209 \mathrm{WIDTH}(\mathrm{Y})=1.364 \mathrm{RESP}(\mathrm{Y})=1.0000 \mathrm{HSQC}=\mathrm{C} 6$

$2-\mathrm{H} 7 \quad / 2 \quad 4.453612 \quad 1 * 1 * 1 \quad \mathrm{STAT}=\mathrm{Y} \quad \mathrm{PRED}=3.489 \mathrm{RANGE}=0.999 \mathrm{WIDTH}(\mathrm{Y})=2.234 \mathrm{RESP}(\mathrm{Y})=1.0000 \mathrm{HSQC}=\mathrm{C} 7$

2-H8 $/ 2 \quad 4.095498 \quad 1 * 1 * 1 \quad$ STAT $=\mathrm{Y} \quad \mathrm{PRED}=3.267 \mathrm{RANGE}=0.519 \mathrm{WIDTH}(\mathrm{Y})=1.980 \mathrm{RESP}(\mathrm{Y})=1.0000 \mathrm{HSQC}=\mathrm{C} 8$

$2-\mathrm{H} 12 / 2 \quad 7.038228 \quad 1 * 1 * 1 \quad \mathrm{STAT}=\mathrm{Y} \quad \mathrm{PRED}=7.238 \mathrm{RANGE}=0.209 \mathrm{WIDTH}(\mathrm{Y})=1.860 \mathrm{RESP}(\mathrm{Y})=1.0000 \mathrm{HSQC}=\mathrm{C} 12$

$2-\mathrm{H} 13 / 2 \quad 6.819431 \quad 1 * 1 * 1 \quad$ STAT $=\mathrm{Y} \quad \mathrm{PRED}=6.798 \mathrm{RANGE}=0.189 \mathrm{WIDTH}(\mathrm{Y})=2.932 \mathrm{RESP}(\mathrm{Y})=1.0000 \mathrm{HSQC}=\mathrm{C} 13$

$2-\mathrm{H} 16 / 2 \quad 7.163894 \quad 1 * 1 * 1 \quad \mathrm{STAT}=\mathrm{Y} \quad \mathrm{PRED}=6.872 \mathrm{RANGE}=0.386 \mathrm{WIDTH}(\mathrm{Y})=1.847 \mathrm{RESP}(\mathrm{Y})=1.0000 \mathrm{HSQC}=\mathrm{C} 16$

$2-\mathrm{H} 26 / 2 \quad 6.108187 \quad 1 * 1 * 1 \quad \mathrm{STAT}=\mathrm{Y} \quad \mathrm{PRED}=5.851 \mathrm{RANGE}=0.366 \mathrm{WIDTH}(\mathrm{Y})=1.256 \mathrm{RESP}(\mathrm{Y})=1.0000 \mathrm{HSQC}=\mathrm{C} 26$

$2-\mathrm{H} 29 / 2 \quad 5.257002 \quad 1 * 1 * 1 \quad \mathrm{STAT}=\mathrm{Y} \quad \mathrm{PRED}=5.846 \mathrm{RANGE}=0.379 \mathrm{WIDTH}(\mathrm{Y})=2.770 \mathrm{RESP}(\mathrm{Y})=1.0000 \mathrm{HSQC}=\mathrm{C} 29$

$2-\mathrm{H} 30 / 2 \quad 3.943025 \quad 1 * 1 * 1 \quad \mathrm{STAT}=\mathrm{Y} \quad \mathrm{PRED}=4.173 \mathrm{RANGE}=0.556 \mathrm{WIDTH}(\mathrm{Y})=2.380 \mathrm{RESP}(\mathrm{Y})=1.0000 \mathrm{HSQC}=\mathrm{C} 30$

$2-\mathrm{H} 31 / 2 \quad 4.676636 \quad 1 * 1 * 1 \quad \mathrm{STAT}=\mathrm{Y} \quad \mathrm{PRED}=2.842 \mathrm{RANGE}=1.289 \mathrm{WIDTH}(\mathrm{Y})=2.672 \operatorname{RESP}(\mathrm{Y})=1.0000 \mathrm{HSQC}=\mathrm{C} 31$

$2-\mathrm{H} 33 / 2 \quad 7.091426 \quad 1 * 1 * 1 \quad \mathrm{STAT}=\mathrm{Y} \quad \mathrm{PRED}=6.776 \mathrm{RANGE}=0.279 \mathrm{WIDTH}(\mathrm{Y})=1.956 \mathrm{RESP}(\mathrm{Y})=1.0000 \mathrm{HSQC}=\mathrm{C} 33$

$2-\mathrm{H} 36 / 2 \quad 6.782072 \quad 1 * 1 * 1 \quad \mathrm{STAT}=\mathrm{Y} \quad \mathrm{PRED}=6.726 \mathrm{RANGE}=0.159 \mathrm{WIDTH}(\mathrm{Y})=1.835 \mathrm{RESP}(\mathrm{Y})=1.0000 \mathrm{HSQC}=\mathrm{C} 36$

$2-\mathrm{H} 37 / 2 \quad 6.928103 \quad 1 * 1 * 1 \quad$ STAT $=Y \quad \mathrm{PRED}=7.242 \mathrm{RANGE}=0.549 \mathrm{WIDTH}(\mathrm{Y})=2.114 \mathrm{RESP}(\mathrm{Y})=1.0000 \mathrm{HSQC}=\mathrm{C} 37$

$2-\mathrm{H} 46 / 2 \quad 5.905540 \quad 1 * 1 * 1 \quad \mathrm{STAT}=\mathrm{Y} \quad \mathrm{PRED}=5.966 \mathrm{RANGE}=0.598 \mathrm{WIDTH}(\mathrm{Y})=1.787 \mathrm{RESP}(\mathrm{Y})=1.0000 \mathrm{HSQC}=\mathrm{C} 46$

$2-\mathrm{H} 49 / 2 \quad 4.964448 \quad 1 * 1 * 1 \quad$ STAT $=\mathrm{Y} \quad \mathrm{PRED}=4.657 \mathrm{RANGE}=0.339 \mathrm{WIDTH}(\mathrm{Y})=3.368 \mathrm{RESP}(\mathrm{Y})=1.0000 \mathrm{HSQC}=\mathrm{C} 49$

$2-\mathrm{H} 50 / 2 \quad 4.271833 \quad 1 * 1 * 1 \quad \mathrm{STAT}=\mathrm{Y} \quad \mathrm{PRED}=3.972 \mathrm{RANGE}=0.289 \mathrm{WIDTH}(\mathrm{Y})=3.101 \mathrm{RESP}(\mathrm{Y})=1.0000 \mathrm{HSQC}=\mathrm{C} 50$

$2-\mathrm{H} 51 \mathrm{~A} / 2 \quad 2.796131 \quad 1 * 1 * 1 \quad \mathrm{STAT}=\mathrm{Y} \quad \mathrm{PRED}=2.696 \mathrm{RANGE}=0.179 \mathrm{WIDTH}(\mathrm{Y})=3.287 \mathrm{RESP}(\mathrm{Y})=1.0000 \mathrm{HSQC}=\mathrm{C} 51$

$2-\mathrm{H} 51 \mathrm{~B} / 2 \quad 2.934165 \quad 1 * 1 * 1 \quad \mathrm{STAT}=\mathrm{Y} \quad \mathrm{PRED}=2.768 \mathrm{RANGE}=0.199 \mathrm{WIDTH}(\mathrm{Y})=2.746 \mathrm{RESP}(\mathrm{Y})=1.0000 \mathrm{HSQC}=\mathrm{C} 51$

$2-\mathrm{H} 53 / 2 \quad 7.112068 \quad 1 * 1 * 1 \quad \mathrm{STAT}=\mathrm{Y} \quad \mathrm{PRED}=6.635 \mathrm{RANGE}=0.508 \mathrm{WIDTH}(\mathrm{Y})=2.170 \mathrm{RESP}(\mathrm{Y})=1.0000 \mathrm{HSQC}=\mathrm{C} 53$

$2-\mathrm{H} 56 / 2 \quad 6.752720 \quad 1 * 1 * 1 \quad \mathrm{STAT}=\mathrm{Y} \quad \mathrm{PRED}=6.666 \mathrm{RANGE}=0.669 \mathrm{WIDTH}(\mathrm{Y})=1.488 \mathrm{RESP}(\mathrm{Y})=1.0000 \mathrm{HSQC}=\mathrm{C} 56$

$2-\mathrm{H} 57 / 2 \quad 6.887189 \quad 1 * 1 * 1 \quad$ STAT $=Y \quad$ PRED $=7.072$ RANGE $=0.399 \mathrm{WIDTH}(\mathrm{Y})=2.265$ RESP(Y)=1.0000 HSQC $=\mathrm{C} 57$

COUPLING CONSTANTS(HZ)

\begin{tabular}{|c|c|c|c|c|c|}
\hline J164_65 & 2.3393 & J 1-H2 & 1-H6 & $\mathrm{STAT}=\mathrm{N}$ & $\mathrm{PRED}=2.230 \mathrm{RANGE}=0.890$ \\
\hline $\mathrm{J} 166 \_67$ & 3.4327 & J 1-H7 & 1-H8 & $\mathrm{STAT}=\mathrm{N}$ & $\mathrm{PRED}=3.670 \mathrm{RANGE}=2.800$ \\
\hline $\mathrm{J} 168-69$ & 8.2689 & J 1-H12 & 1-H13 & $\mathrm{STAT}=\mathrm{N}$ & $\mathrm{PRED}=8.260 \mathrm{RANGE}=0.500$ \\
\hline $\mathrm{J} 168-70$ & 2.1735 & J $1-\mathrm{H} 12$ & $1-\mathrm{H} 16$ & $\mathrm{STAT}=\mathrm{N}$ & $\mathrm{PRED}=2.030 \mathrm{RANGE}=0.800$ \\
\hline $\mathrm{J} 169-70$ & 0.0037 & J $1-\mathrm{H} 13$ & 1-H16 & $\mathrm{STAT}=\mathrm{N}$ & $\mathrm{PRED}=0.430 \mathrm{RANGE}=0.320$ \\
\hline $\mathrm{J} 177_{-}^{-} 78$ & 0.7847 & J 1-H29 & 1-H30 & $\mathrm{STAT}=\mathrm{N}$ & $\mathrm{PRED}=1.060 \mathrm{RANGE}=2.000$ \\
\hline $178 \_79$ & 1.9396 & J $1-\mathrm{H} 30$ & $1-\mathrm{H} 31$ & $\mathrm{STAT}=\mathrm{N}$ & $\mathrm{PRED}=1.590 \mathrm{RANGE}=2.200$ \\
\hline $\mathrm{J} 180 \_81$ & 0.0002 & J 1-H33 & 1-H36 & $\mathrm{STAT}=\mathrm{N}$ & $\mathrm{PRED}=0.430 \mathrm{RANGE}=0.320$ \\
\hline $\mathrm{J} 180 \_82$ & 2.0838 & J 1-H33 & 1-H37 & $\mathrm{STAT}=\mathrm{N}$ & $\mathrm{PRED}=2.030 \mathrm{RANGE}=0.800$ \\
\hline $\mathrm{J} 18182$ & 8.1893 & J 1-H36 & 1-H37 & $\mathrm{STAT}=\mathrm{N}$ & $\mathrm{PRED}=8.260 \mathrm{RANGE}=0.500$ \\
\hline $\mathrm{J} 187^{-} 88$ & 0.0994 & J 1-H49 & 1-H50 & $\mathrm{STAT}=\mathrm{N}$ & $\mathrm{PRED}=1.140 \mathrm{RANGE}=2.200$ \\
\hline J188_89 & & J 1-H50 & 1-H51A & $\mathrm{STAT}=\mathrm{N}$ & $\mathrm{PRED}=2.010 \mathrm{RANGE}=2.200$ \\
\hline $\mathrm{J} 188 \_90$ & 4.8605 & J 1-H50 & 1-H51B & $\mathrm{STAT}=\mathrm{N}$ & $\mathrm{PRED}=4.410 \mathrm{RANGE}=2.800$ \\
\hline $\mathrm{J} 189-90$ & -17.3564 & J 1-H51A & 1-H51B & $\mathrm{STAT}=\mathrm{N}$ & $\mathrm{PRED}=-14.870 \mathrm{RANGE}=1.280$ \\
\hline J191_92 & 0.0266 & J 1-H53 & 1-H56 & $\mathrm{STAT}=\mathrm{N}$ & $\mathrm{PRED}=0.430 \mathrm{RANGE}=0.320$ \\
\hline J191_93 & 2.0260 & J 1-H53 & 1-H57 & $\mathrm{STAT}=\mathrm{N}$ & $\mathrm{PRED}=2.030 \mathrm{RANGE}=0.800$ \\
\hline $\mathrm{J} 192 \_93$ & 8.1594 & J 1-H56 & 1-H57 & $\mathrm{STAT}=\mathrm{N}$ & $\mathrm{PRED}=8.260 \mathrm{RANGE}=0.500$ \\
\hline $\mathrm{J} 264^{-} 65$ & 2.3361 & J 2-H2 & 2-H6 & $\mathrm{STAT}=\mathrm{N}$ & $\mathrm{PRED}=2.230 \mathrm{RANGE}=0.890$ \\
\hline $\mathrm{J} 266 \_67$ & 3.4319 & J 2-H7 & 2-H8 & $\mathrm{STAT}=\mathrm{N}$ & $\mathrm{PRED}=3.670 \mathrm{RANGE}=2.800$ \\
\hline $\mathrm{J} 268 \_69$ & 8.2611 & J 2-H12 & 2-H13 & $\mathrm{STAT}=\mathrm{N}$ & $\mathrm{PRED}=8.260 \mathrm{RANGE}=0.500$ \\
\hline $\mathrm{J} 268 \_70$ & 2.1671 & J 2-H12 & 2-H16 & $\mathrm{STAT}=\mathrm{N}$ & $\mathrm{PRED}=2.030 \mathrm{RANGE}=0.800$ \\
\hline $\mathrm{J} 269-70$ & 0.0166 & J 2-H13 & 2-H16 & $\mathrm{STAT}=\mathrm{N}$ & $\mathrm{PRED}=0.430 \mathrm{RANGE}=0.320$ \\
\hline $\mathrm{J} 277_{-}^{-} 78$ & 0.5632 & J 2-H29 & 2-H30 & $\mathrm{STAT}=\mathrm{N}$ & $\mathrm{PRED}=1.060 \mathrm{RANGE}=2.000$ \\
\hline $\mathrm{J} 278 \_79$ & 1.9340 & J 2-H30 & 2-H31 & $\mathrm{STAT}=\mathrm{N}$ & $\mathrm{PRED}=1.590 \mathrm{RANGE}=2.200$ \\
\hline $\mathrm{J} 280 \_81$ & 0.5591 & J 2-H33 & 2-H36 & $\mathrm{STAT}=\mathrm{N}$ & $\mathrm{PRED}=0.430 \mathrm{RANGE}=0.320$ \\
\hline
\end{tabular}




\begin{tabular}{|c|c|c|c|c|c|}
\hline J280_82 & 2.2472 & J $2-\mathrm{H} 33$ & 2-H37 & $\mathrm{STAT}=\mathrm{N}$ & $\mathrm{PRED}=2.030 \mathrm{RANGE}=0.800$ \\
\hline J281_82 & 8.2358 & J 2-H36 & 2-H37 & $\mathrm{STAT}=\mathrm{N}$ & $\mathrm{PRED}=8.260 \mathrm{RANGE}=0.500$ \\
\hline J287_88 & 0.0022 & J 2-H49 & 2-H50 & $\mathrm{STAT}=\mathrm{N}$ & $\mathrm{PRED}=1.140 \mathrm{RANGE}=2.200$ \\
\hline J288_89 & 3.2159 & J 2-H50 & 2-H51A & $\mathrm{STAT}=\mathrm{N}$ & $\mathrm{PRED}=2.010 \mathrm{RANGE}=2.200$ \\
\hline J288_90 & 4.7611 & J $2-\mathrm{H} 50$ & $2-\mathrm{H} 51 \mathrm{~B}$ & $\mathrm{STAT}=\mathrm{N}$ & $\mathrm{PRED}=4.410 \mathrm{RANGE}=2.800$ \\
\hline $\mathrm{J} 28990$ & -17.3652 & J 2-H51A & 2-H51B & $\mathrm{STAT}=\mathrm{N}$ & $\mathrm{PRED}=-14.870 \mathrm{RANGE}=1.280$ \\
\hline J291_92 & 0.3464 & J 2-H53 & 2-H56 & $\mathrm{STAT}=\mathrm{N}$ & $\mathrm{PRED}=0.430 \mathrm{RANGE}=0.320$ \\
\hline J291_93 & 2.0125 & J 2-H53 & 2-H57 & $\mathrm{STAT}=\mathrm{N}$ & $\mathrm{PRED}=2.030 \mathrm{RANGE}=0.800$ \\
\hline J292_93 & 8.1644 & J 2-H56 & 2-H57 & $\mathrm{STAT}=\mathrm{N}$ & $\mathrm{PRED}=8.260 \mathrm{RANGE}=0.500$ \\
\hline
\end{tabular}

CONTROL PARAMETERS:

Solvent $=$ none $($ def. $99 \%$ enriched $)$

$1.000=$ Concentration $($ vol $\%$, def $=1.0 \%)$

$0.00100000=$ Minimum line-intensity

$0.00100000=$ Diagonalization criterium (not in use)

$899.93600000=$ FIELD $(1 \mathrm{H}, \mathrm{MHz})$, used to transform shifts to ppms

$19.92595689=$ Left frequency $(\mathrm{ppm})$

$-10.18757232=$ Right frequency $(\mathrm{ppm})$

$10.000=$ Acquisition time (s, for QMTLS)

$0.000=$ Line-width (for modes D, P \& T, $0=$ use defaults)

$0.206760153=$ Data-point resolution $(\mathrm{Hz})$

$31.203=$ GAUSSIAN $(\%, 0=$ use default from INF $)$

$-0.921=$ Dispersion contribution $(\%, 0=$ use default from INF)

$0.00000000=$ Decoupling frequency (for DORES) 
S24. The CD spectra of compounds 1-3.

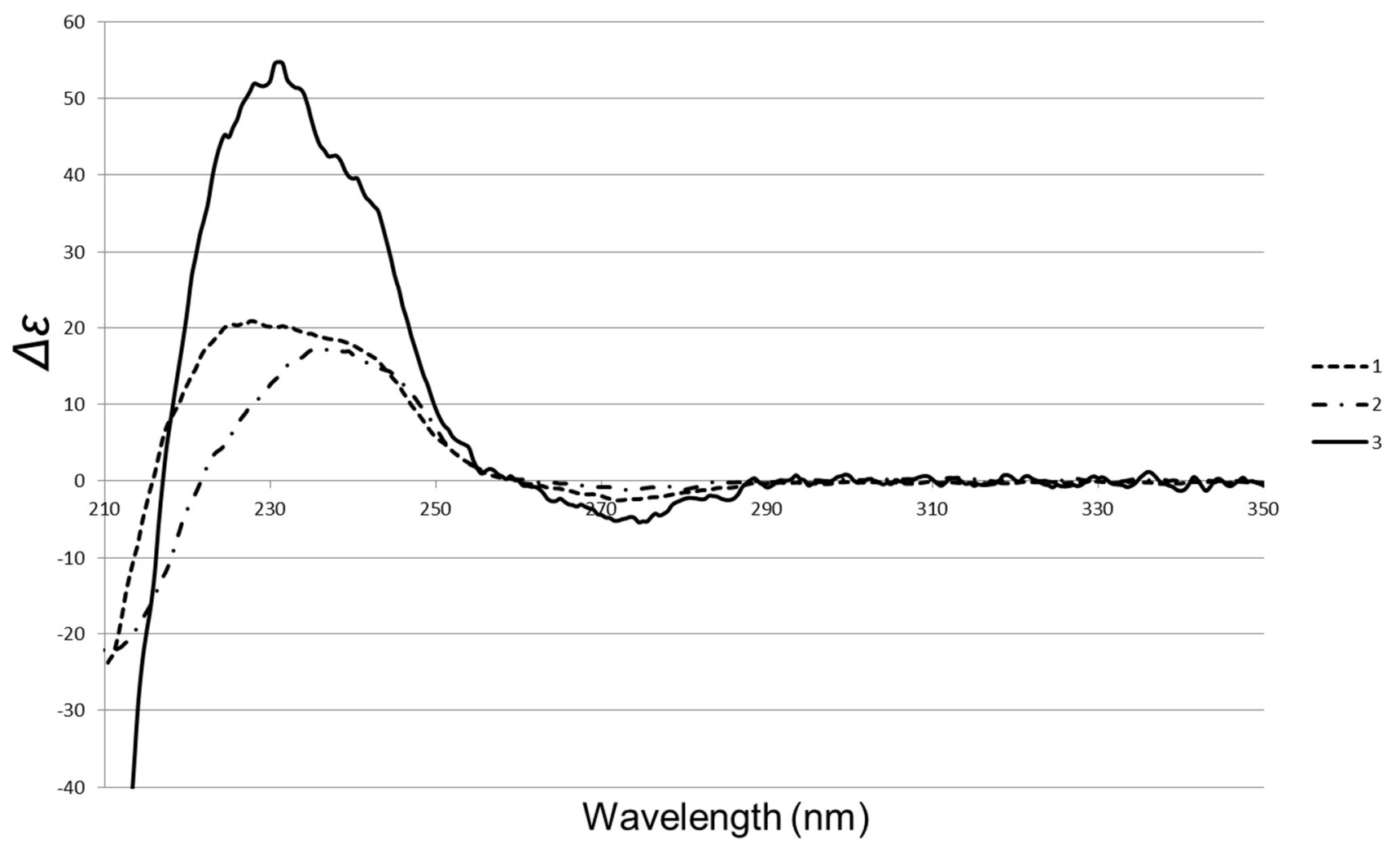


S25. ${ }^{1} \mathrm{H}$ Comparison of NMR spectra showing that deuterium exchange reaction occurred in 2.

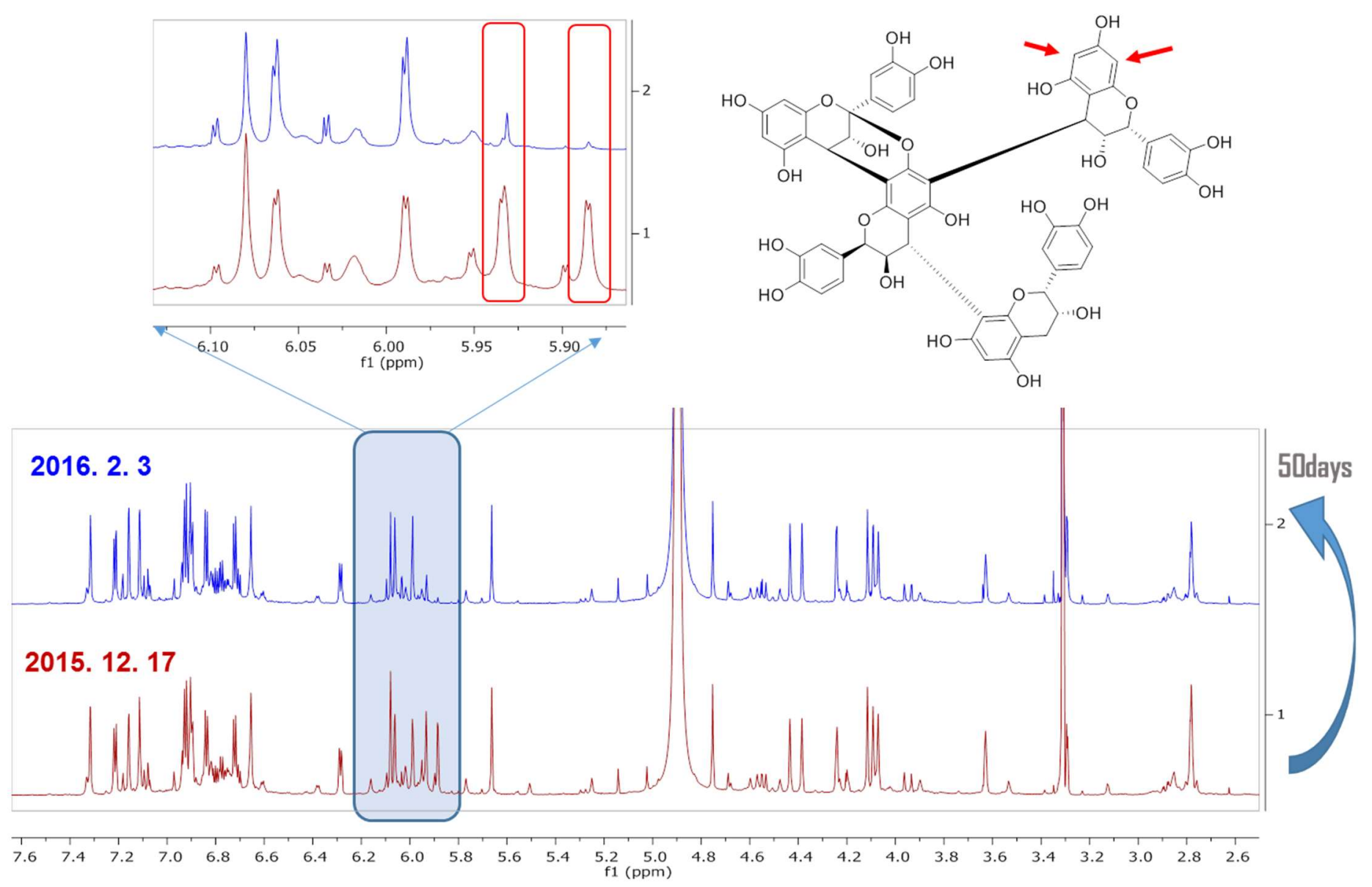

MeOD, 298K, $900 \mathrm{MHz}$, stored in $\operatorname{MeOD}\left(-20^{\circ} \mathrm{C}\right)$ for 50 days 
S26. Calibration of the NMR acquisition temperature.

\section{$\square$ VT-Calibration_800 MHz}

$170 \mu \mathrm{l}, 3 \mathrm{~mm}$ tube

(with non-deuterated solvents)

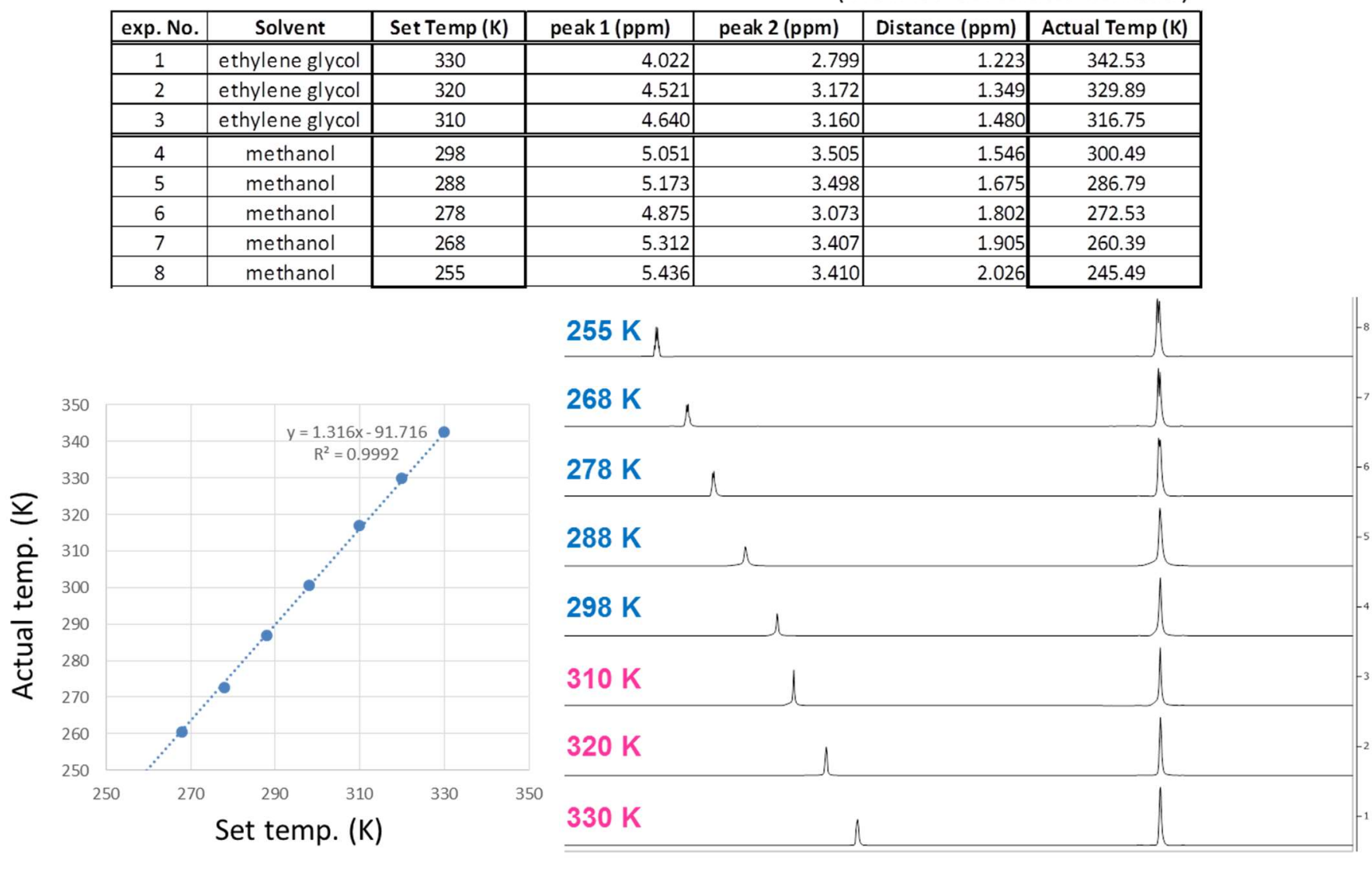

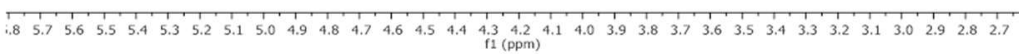


S27. Calculation of the purity of $\mathbf{1}$ by qHNMR using the $100 \%$ method.

\begin{tabular}{|c|c|}
\hline Composition & Weight\% \\
\hline Major & 71.80 \\
\hline Minor 1 & 21.67 \\
\hline Minor 2 & 0.92 \\
\hline Acetic acid & 0.02 \\
\hline Acetonitrile & 0.24 \\
\hline Formic acid & 5.35 \\
\hline
\end{tabular}

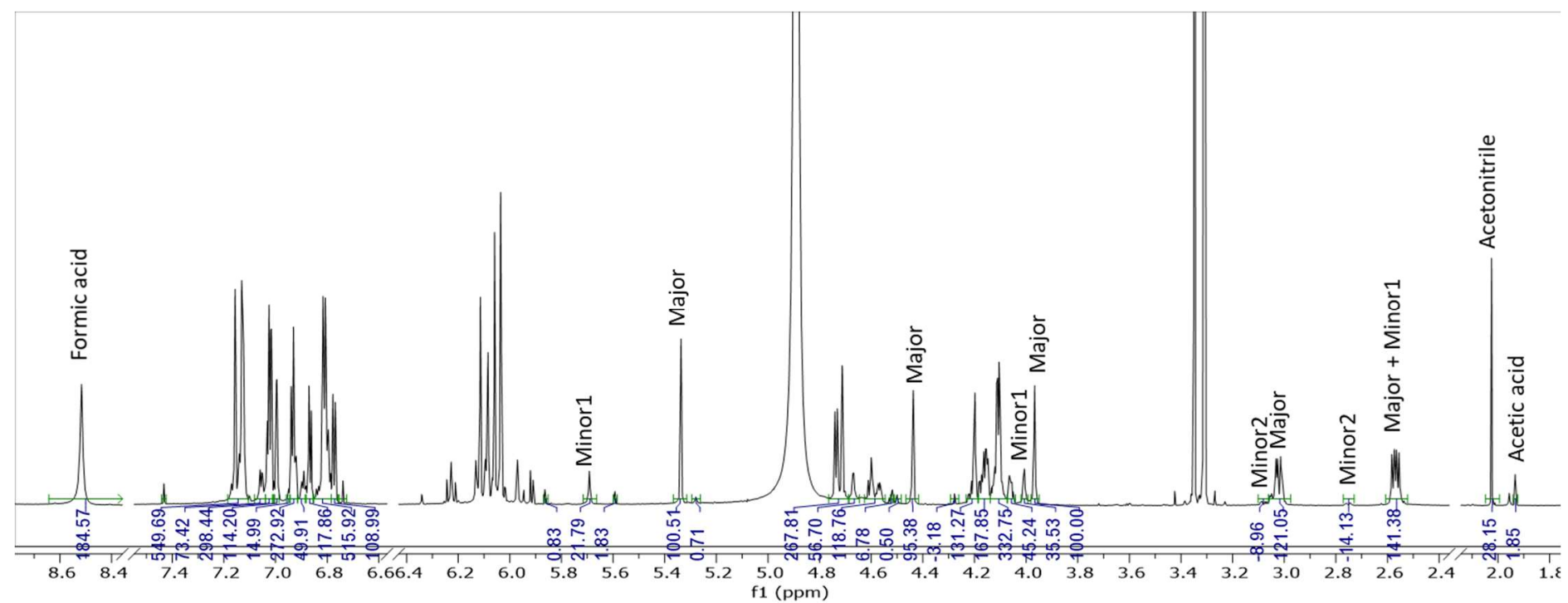

Cleanest integrals used for quantitation are labeled. The signals for $\mathrm{H}-6 \mathrm{~s}$ and $\mathrm{H}-8 \mathrm{~s}$ of the $\mathrm{A}$ rings are excluded from the calculation because of the $H-D$ exchange reaction.

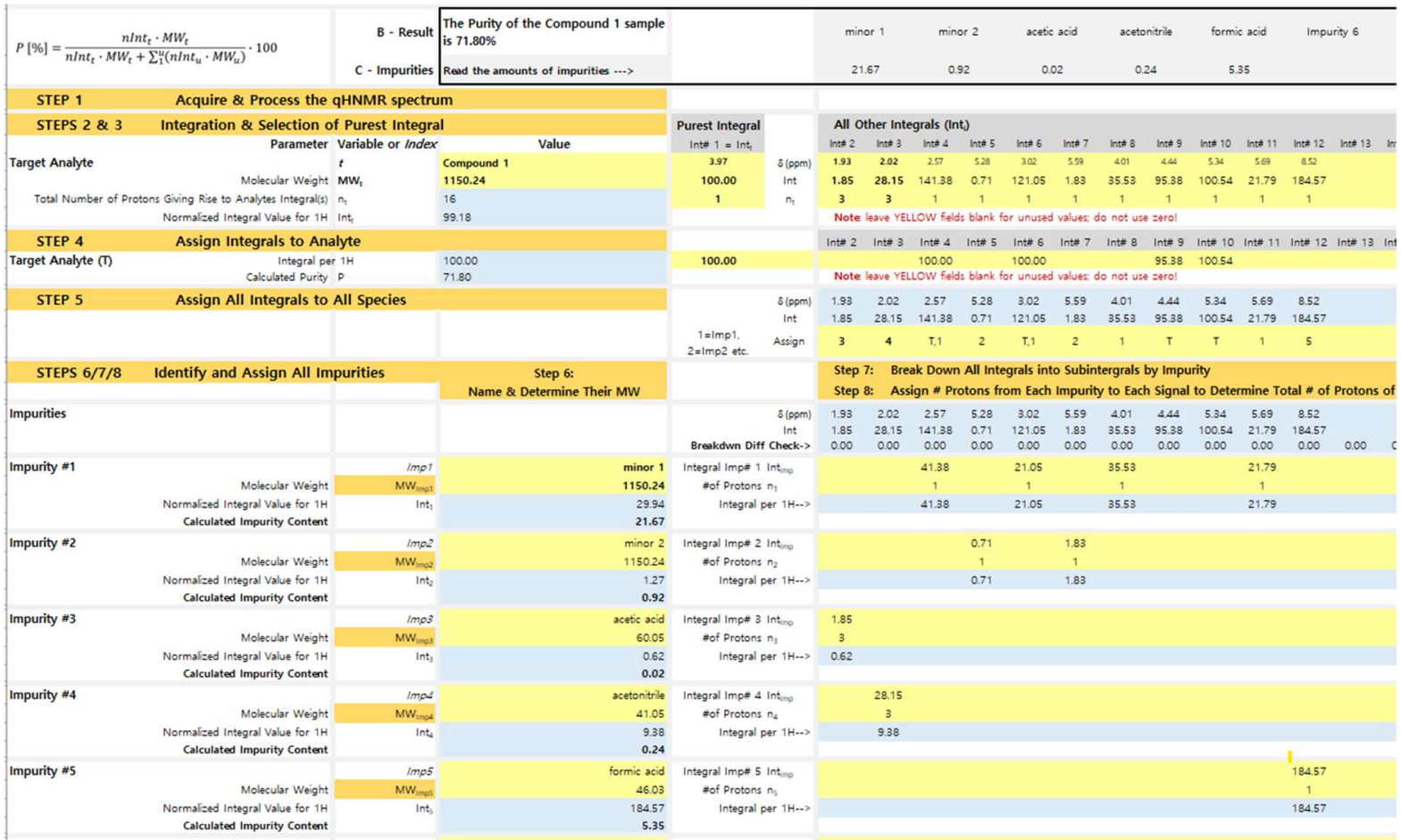

Spreadsheet Calculations for qHNMR Analysis GF Pauli et al., J. Med. Chem. 2014, 57, 22, 9220-9231 
S28. Calculation of the purity of 2 by qHNMR using the $100 \%$ method.

\begin{tabular}{|c|c|}
\hline Composition & Weight\% \\
\hline Major & 69.40 \\
\hline Minor & 19.18 \\
\hline Ethanol & 0.05 \\
\hline Grease & 0.08 \\
\hline Acetonitrile & 11.30 \\
\hline
\end{tabular}

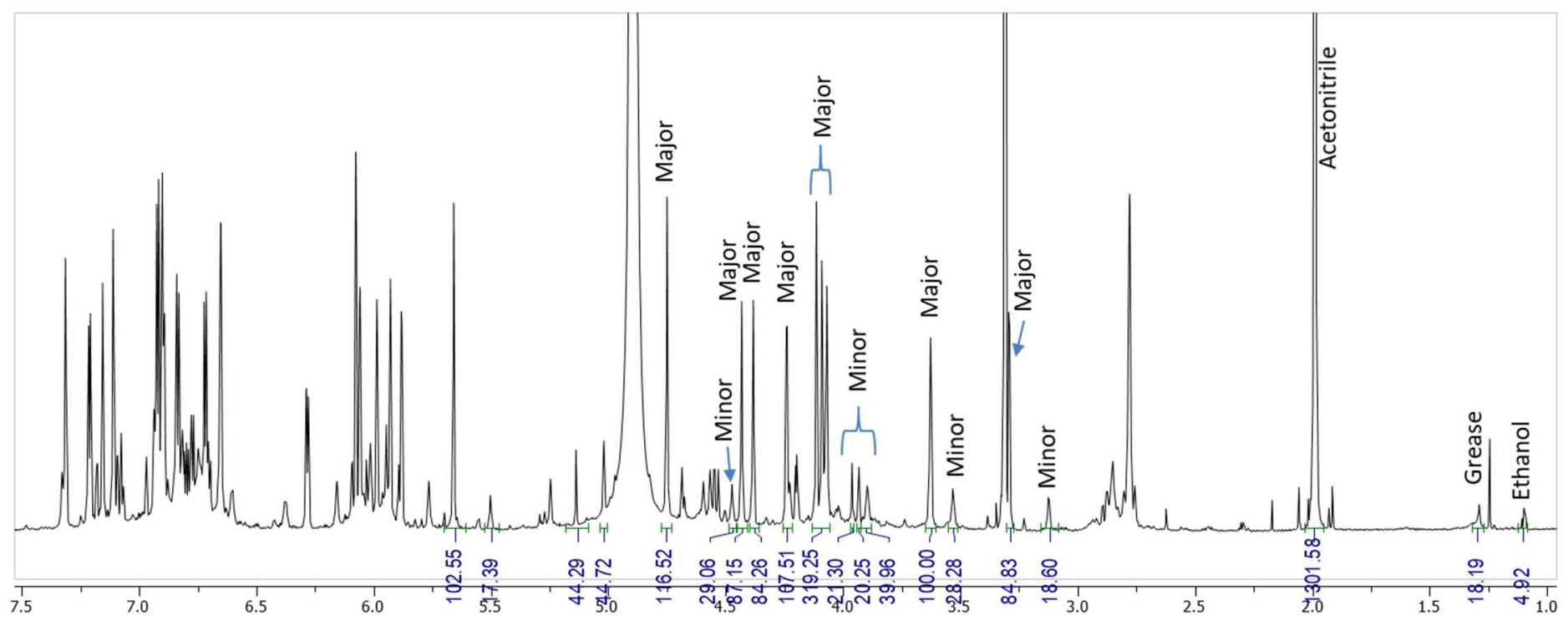

Cleanest integrals used for quantitation are labeled. The signals for $\mathrm{H}-6 \mathrm{~s}$ and $\mathrm{H}-8 \mathrm{~s}$ of the $\mathrm{A}$ rings are excluded from the calculation because of the $H-D$ exchange reaction.

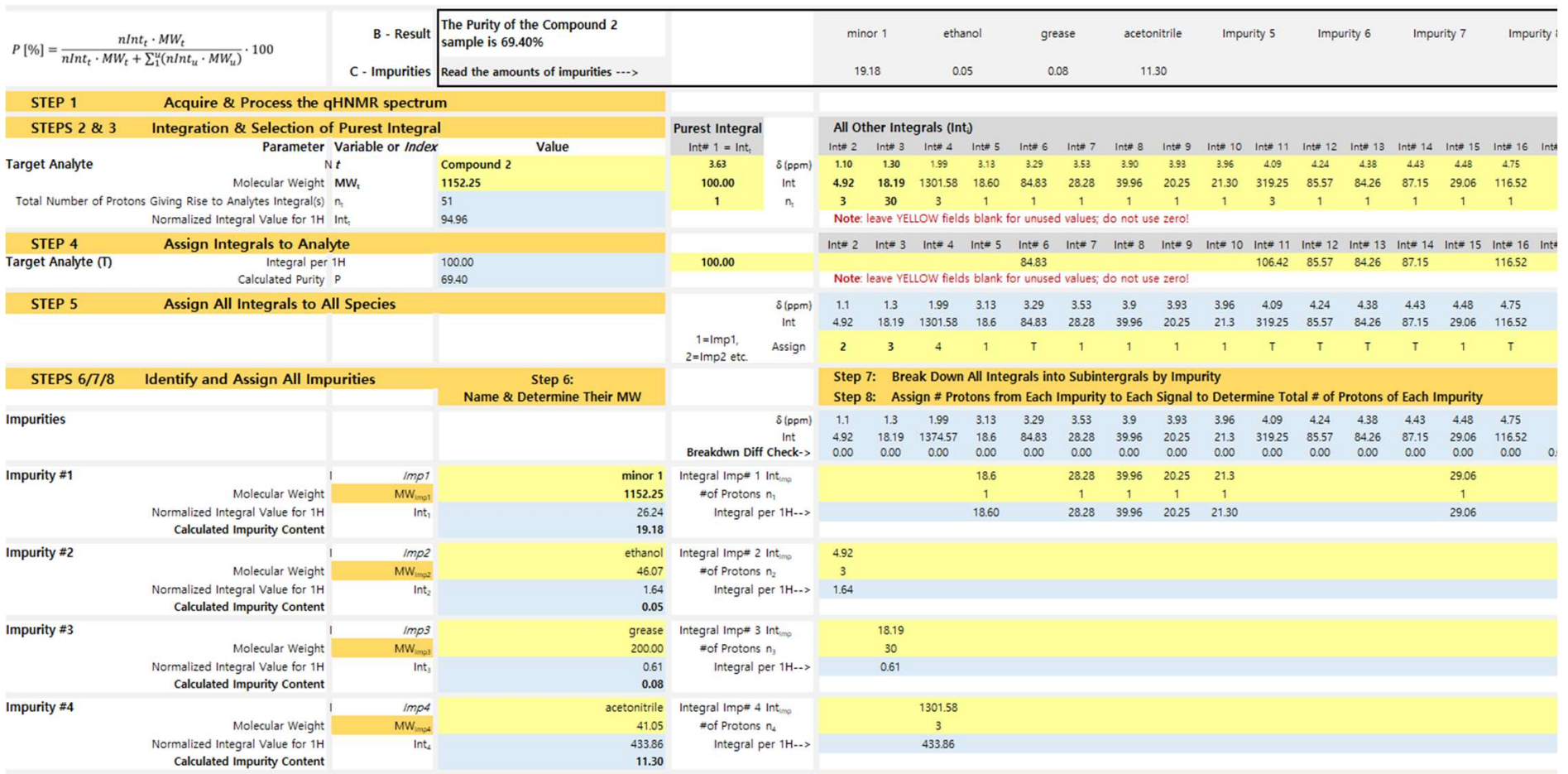

Spreadsheet Calculations for qHNMR Analysis GF Pauli et al., J. Med. Chem. 2014, 57, 22, 9220-9231 
S29. Calculation of the purity of $\mathbf{3}$ by qHNMR using the $100 \%$ method.

\begin{tabular}{|c|c|}
\hline Composition & Weight\% \\
\hline Major & 70.32 \\
\hline Minor & 20.01 \\
\hline Formic acid & 9.37 \\
\hline Acetonitrile & 0.32 \\
\hline
\end{tabular}

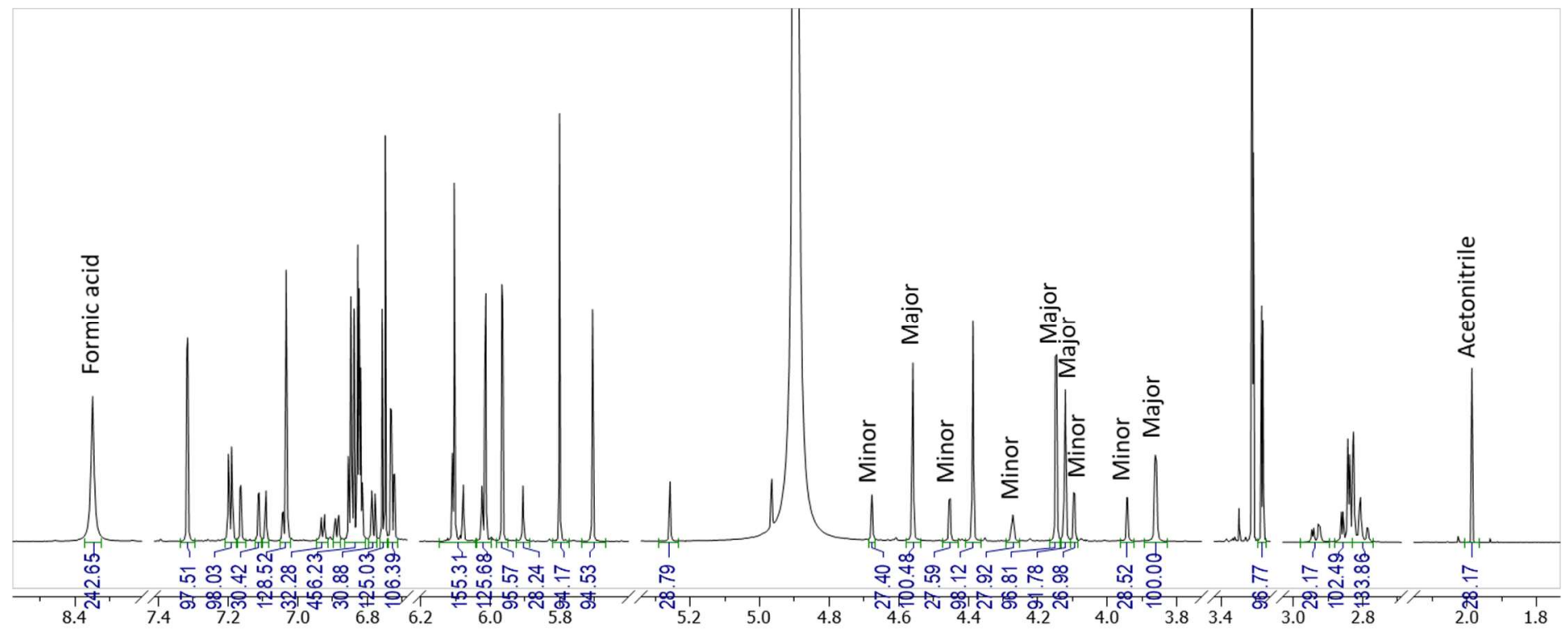

Cleanest integrals used for quantitation are labeled. The signals for $\mathrm{H}-6 \mathrm{~s}$ and $\mathrm{H}-8 \mathrm{~s}$ of the A rings are excluded from the calculation because of the $H-D$ exchange reaction.

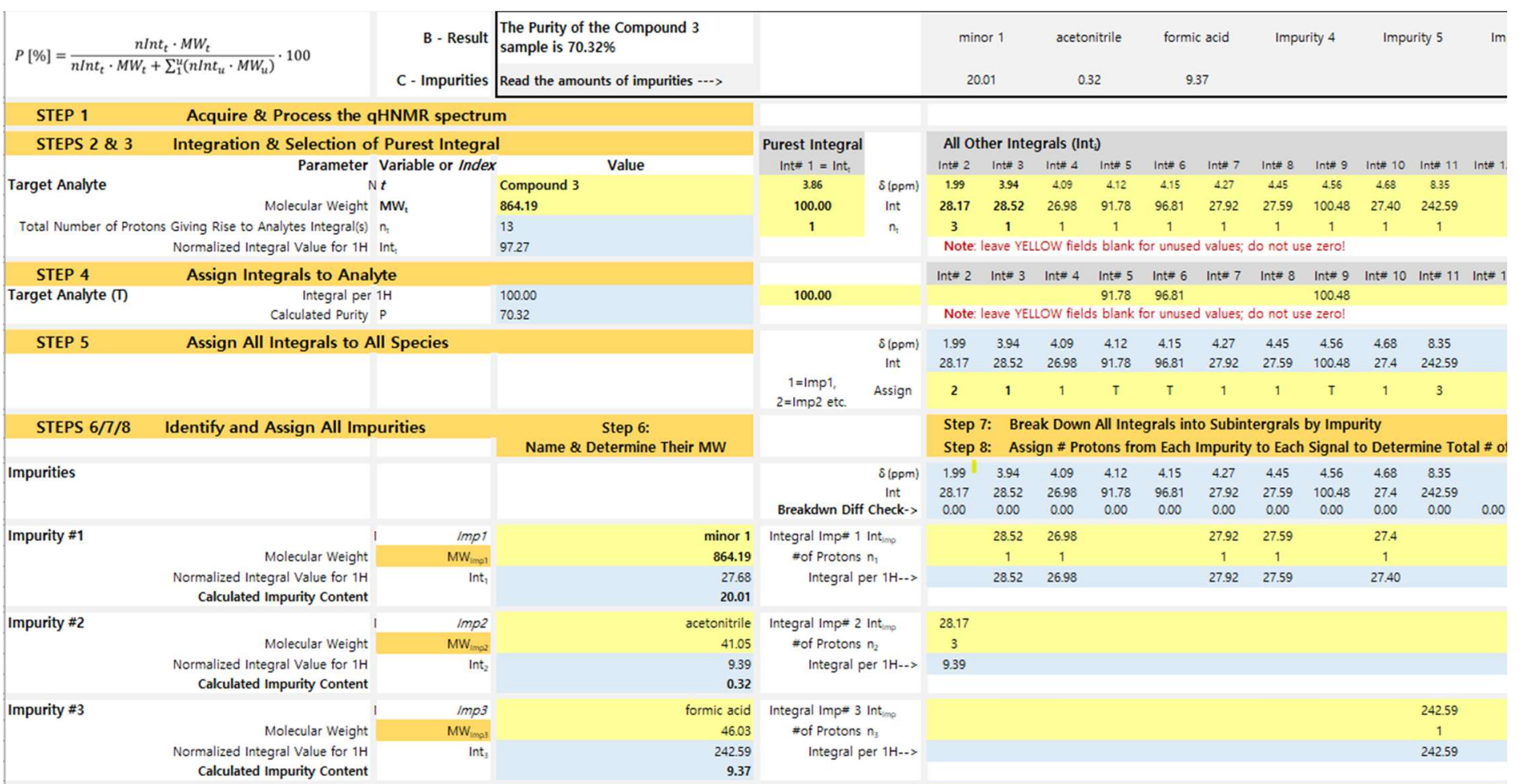

Spreadsheet Calculations for qHNMR Analysis GF Pauli et al., J. Med. Chem. 2014, 57, 22, 9220-9231 